\title{
The Strong Influence of Substrate Conductivity on Droplet
}

\section{Evaporation}

\author{
G. J. DUN $N^{1}$, S. K. WIL SON ${ }^{1}$, B. R. DUFFY $Y^{1}$, S. DA VID AND K. SEFIA NE \\ ${ }^{1}$ Department of Mathematics, University of Strathclyde, Livingstone Tower, 26 Richmond Street, Glasgow, G1 1XH \\ ${ }^{2}$ School of Engineering and Electronics, The University of Edinburgh, The King's Buildings, Mayfield Road, Edinburgh, EH9
} $3 \mathrm{JL}$

(Received 22nd December 2007, revised 31st May, 4th November and 17th November 2008)

We report the results of physical experiments that demonstrate the strong influence of the thermal conductivity of the substrate on the evaporation of a pinned droplet. We show that this behaviour can be captured by a mathematical model including the variation of the saturation concentration with temperature, and hence coupling the problems for the vapour concentration in the atmosphere and the temperature in the liquid and the substrate. Furthermore, we show that including two ad hoc improvements to the model, namely a Newton's law of cooling on the unwetted surface of the substrate and the buoyancy of water vapour in the atmosphere, gives excellent quantitative agreement for all of the combinations of liquid and substrate considered.

\section{Introduction}

In recent years there has been a rapid growth of interest in the technologically important and scientifically interesting problem of the evaporation of liquid droplets. In what follows we do not attempt to give a comprehensive review of this rapidly expanding field, but simply highlight some of the key papers relevant to the subject of the present work, namely the evaporation of pinned droplets (i.e. droplets whose contact lines are fixed on a substrate).

More than thirty years ago Picknett \& Bexon (1977) studied the evaporation of sessile droplets both ex- 
perimentally and theoretically. Experimentally they identified two extreme modes of evaporation, namely a constant-contact-angle mode and a constant-contact-area (i.e. constant droplet radius) mode. They also proposed a theoretical model (hereafter referred to as "the basic model" for simplicity) based on the assumption that the atmosphere just above the free surface of the droplet is saturated with vapour and that diffusion of liquid vapour away from the droplet is the rate-limiting evaporative process (see, for example, Poulard, Guéna \& Cazabat (2005) and Popov (2005)). By using the known exact solution for the electrical potential of a charged lens-shaped conductor (see, for example, Lebedev (1965)), Picknett \& Bexon (1977) obtained both the exact and an accurate approximate solution for the total mass flux from the free surface of the droplet. Subsequently Bourgès-Monnier \& Shanahan (1995) conducted more accurate experiments and identified four distinct stages to the evaporation process; they also obtained an approximate solution for the total mass flux from the free surface of the droplet. Deegan et al. (1997) and Deegan et al. (2000) developed a theoretical model for the evaporation of a pinned liquid droplet which, in particular, shows how evaporation drives a radially outwards flow within the droplet which can cause any dispersed solids to form a ring stain near the contact line as the droplet dries (the so-called "coffee-stain problem"). Hu \& Larson (2002) performed numerical computations using a finite-element method to obtain a simple approximation to the total mass flux from the droplet as a function of the contact angle. Subsequently Hu \& Larson (2005a) undertook further numerical calculations in order to investigate the flow within the droplet, and Hu \& Larson (2005b) generalised this analysis to include thermocapillary (Marangoni) effects. In particular, Hu \& Larson (2005b) found that at larger contact angles thermocapillary effects drive a recirculation flow within the droplet, whereas at smaller contact angles the flow is always radially outwards (as it is in the absence of thermocapillary effects). Subsequently Hu \& Larson (2006) showed both experimentally and theoretically that sufficiently strong thermocapillary effects can suppress the formation of a ring stain as a droplet containing dispersed solids evaporates. Savino, Paterna \& Favaloro (2002) investigated both experimentally and numerically the influence of buoyancy and thermocapillary effects 
on the evaporation of a pendant droplet hanging from a disk held at a constant temperature. Recently both Xu \& Luo (2007) and Ristenpart et al. (2007) observed recirculation driven by thermocapillary effects experimentally, and Ristenpart et al. (2007) showed that the occurrence of recirculation depends on the relative thermal conductivities of the liquid and the substrate as well as the contact angle.

All of the theoretical work described above is based on the assumption that the atmosphere just above the free surface of the droplet is saturated with vapour and that diffusion of liquid vapour away from the droplet is the rate-limiting evaporative process. There is another body of work (including that by Burelbach, Bankoff \& Davis (1988), Anderson \& Davis (1995), Ajaev (2005) and Sodtke, Ajaev \& Stephan (2007)) concerning the rather different situation in which diffusion of vapour can be neglected and nonequilibrium effects at the free surface of the droplet are the rate-limiting evaporative process. In this situation the local mass flux is usually determined from classical kinetic theory. However, it should be noted that Fang \& Ward (1999) have conducted physical experiments that exhibit temperature discontinuities at the free surface of an evaporating liquid which are much larger than and in the opposite direction from that predicted by either classical kinetic theory or nonequilibrium thermodynamics (i.e. the temperature in the vapour just above the free surface is higher than that in the liquid just below it).

Recently Sultan, Boudaoud \& Ben Amar (2005) unified the two approaches by formulating a general local conservation-of-mass condition at the free surface of an evaporating liquid which reduces to the diffusion-limited and non-equilibrium-limited conditions in the appropriate limits. Sultan, Boudaoud \& Ben Amar (2005) also generalised the basic model by allowing the saturation concentration of vapour in the atmosphere just above the free surface of the droplet to be a known function of temperature (rather than simply a known constant as had been assumed by previous authors). In particular, Sultan, Boudaoud \& Ben Amar (2005) investigated the linear stability of an evaporating film and found qualitative agreement with the experimental results of Poulard, Bénichou \& Cazabat (2003).

Much of the work described above concerns pinned droplets. However, there has also been work on 
the spreading of unpinned evaporating droplets. Shanahan (2001a) and Shanahan (2001b) proposed and analysed a theoretical model for spreading due to evaporation and condensation in the vicinity of the contact line. In recent years Cazabat and her collaborators have undertaken an extensive programme of experimental work on the spreading of evaporating droplets described by Cachile, Bénichou \& Cazabat (2002), Cachile et al. (2002), Poulard, Bénichou \& Cazabat (2003), Poulard et al. (2005), Poulard, Guéna \& Cazabat (2005), Guéna et al. (2006), Guéna, Allançon \& Cazabat (2007), Guéna, Poulard \& Cazabat (2007a) and Guéna, Poulard \& Cazabat (2007b).

Shahidzadeh-Bonn et al. (2006) also investigated the spreading of evaporating droplets experimentally. In particular, they found anomalous results for water droplets, which they explained by arguing that, because water vapour is lighter than air, buoyant convection of water vapour in the atmosphere enhances diffusion away from a droplet of water. On the other hand, Guéna, Poulard \& Cazabat (2007a) found experimentally that pendant and sessile evaporating droplets of octane and water behave similarly and concluded that buoyant convection of vapour in the atmosphere is therefore not significant. In addition, Sefiane and his collaborators have investigated several aspects of droplet evaporation experimentally, including droplets on a heated substrate (Mollaret et al. (2004)), the de-pinning of droplets on rough substrates (Sefiane \& Tadrist (2006)), and droplets on substrates with differing thermal conductivities (David, Sefiane \& Tadrist (2007)).

Most of the previous experimental work on droplet evaporation (of which a representative selection is given in Table 1) has used various liquids and substrates apparently without properly taking the influence of the differing thermal conductivities of the substrates fully into account. The recent physical experiments by David, Sefiane \& Tadrist (2007) using a variety of liquids on a variety of substrates show that the thermal conductivity of the substrate has a strong influence on the total evaporation rate. This behaviour is not captured by the basic model, and the main purpose of the present work is to show that a suitably generalised version of the basic model including the variation of the saturation concentration with temperature, and 


\section{Authors}

Picknett \& Bexon (1977)

Birdi, Vu \& Winter (1989)

Bourgès-Monnier \& Shanahan (1995)

Rowan, Newton \& McHale (1995)

Chandra et al. (1996)

Bernardin et al. (1997)

Deegan et al. (2000)

Erbil, McHale \& Newton (2002a)

Erbil, McHale \& Newton (2002b)

Panwar, Barthwal \& Ray (2003)

Crafton \& Black (2004)

Poulard, Guéna \& Cazabat (2005)

Grandas et al. (2005)

Shahidzadeh-Bonn et al. (2006)

$$
\text { Liquid(s) Used }
$$

Methyl acetoacetate

Water

Water

$n$-decane

Water

Water with surfactants

Water

Water with a suspension of colloidal particles

$$
\begin{gathered}
n \text {-nonane } \\
n \text {-octane } \\
\text { Toluene } \\
n \text {-butanol } \\
n \text {-butanol } \\
\text { Toluene } \\
n \text {-nonane } \\
n \text {-octane }
\end{gathered}
$$

Water

$$
\begin{gathered}
\text { Water } \\
n \text {-heptane } \\
\text { Water } \\
\text { Hexane } \\
\text { Heptane } \\
\text { Octane }
\end{gathered}
$$

Nonane

Water
Glass

Polycarbonate

Substrate(s) Used

PTFE

Glass

Epoxy Resin

Polyethylene

PTFE

Glass

PMMA

Stainless Steel

Aluminium

Glass

PMMA

PET

Glass

PTFE

Aluminium

Copper

Silicon

Aluminium

PTFE

Mica

\section{Hexane}

TABLE 1. A representative selection of the liquids and substrates used in droplet-evaporation experiments conducted by previous authors. Note that the thermal conductivities of the substrates used range over three orders of magnitude, from approximately 0.2 $\mathrm{kg} \mathrm{m} \mathrm{s}^{-3} \mathrm{~K}^{-1}$ for PMMA to approximately $400 \mathrm{~kg} \mathrm{~m} \mathrm{~s}^{-3} \mathrm{~K}^{-1}$ for copper. 


$\begin{array}{ccccccc}\text { Parameter } & \text { Symbol } & \text { Units } & \text { Aluminium } & \text { Titanium } & \text { Macor } & \text { PTFE } \\ \text { Density of Substrate } & \rho^{\mathrm{s}} & \mathrm{kg} \mathrm{m}^{-3} & 2.71 \times 10^{3} & 4.54 \times 10^{3} & 2.52 \times 10^{3} & 2.20 \times 10^{3} \\ \text { Specific Heat Capacity of Substrate } & c_{\mathrm{p}}^{\mathrm{s}} & \mathrm{m}^{2} \mathrm{~s}^{-2} \mathrm{~K}^{-1} & 913 & 523 & 790 & 1.05 \times 10^{3} \\ \text { Thermal Conductivity of Substrate } & k^{\mathrm{s}} & \mathrm{kg} \mathrm{m} \mathrm{s}^{-3} \mathrm{~K}^{-1} & 237 & 21.9 & 1.46 & 0.25\end{array}$

TABLE 2. Physical properties of the four substrates used at temperature $T_{\mathrm{a}}=295 \mathrm{~K}$ and pressure $p_{\mathrm{a}}=99.8 \mathrm{kPa}$. The values of $\rho^{\mathrm{s}}$ and $c_{\mathrm{p}}^{\mathrm{s}}$ were taken from Tennent (1971) (aluminium, titanium and PTFE) and Corning (2007) (Macor), and the values of $k^{\mathrm{s}}$ were taken from David, Sefiane \& Tadrist (2007).

hence coupling the problems for the vapour concentration in the atmosphere and the temperature in the liquid and the substrate, is capable of quantitatively reproducing and explaining the experimental results. In particular, the present work generalises the authors' recent analysis of the mathematically convenient special case of a thin droplet on a thin substrate (Dunn et al. (2008)) which gave encouraging qualitative agreement with the experimental results, to the larger values of the contact angle observed experimentally.

\section{Experimental Procedure}

The experimental procedure involved depositing a droplet of a pure liquid with a controlled volume onto a substrate and observing its behaviour as it spontaneously evaporated. A schematic diagram illustrating the experimental set-up is shown in Figure 1.

The four different substrates used, namely aluminium (Al), titanium (Ti), Macor (a machinable glass ceramic manufactured by Corning Incorporated) and polytetrafluoroethylene (PTFE), were chosen for their widely differing thermal conductivities. Relevant physical properties of the substrates used are listed in Table 2. All of the substrates had the same physical dimensions of $1 \mathrm{~cm} \times 1 \mathrm{~cm} \times 0.1 \mathrm{~cm}$ (length $\times$ breadth $\times$ thickness). In order to give all of the substrates the same surface free energy (and hence the same wettability properties) without significantly altering their thermal properties, all were coated with a 


\begin{tabular}{|c|c|c|c|c|c|}
\hline Parameter & Symbol & Units & Acetone & Methanol & Water \\
\hline Molar Mass of Liquid & $M$ & $\mathrm{~kg} \mathrm{~mol}^{-1}$ & $58.1 \times 10^{-3}$ & $32.0 \times 10^{-3}$ & $18.0 \times 10^{-3}$ \\
\hline Density of Liquid & $\rho$ & $\mathrm{kg} \mathrm{m}^{-3}$ & 788 & 790 & 998 \\
\hline Latent Heat of Vaporisation of Liquid & $\mathscr{L}$ & $\mathrm{m}^{2} \mathrm{~s}^{-2}$ & $5.49 \times 10^{5}$ & $1.20 \times 10^{6}$ & $2.45 \times 10^{6}$ \\
\hline Viscosity of Liquid & $\mu$ & $\mathrm{kg} \mathrm{m}^{-1} \mathrm{~s}^{-1}$ & $3.15 \times 10^{-4}$ & $5.74 \times 10^{-4}$ & $9.62 \times 10^{-4}$ \\
\hline Specific Heat Capacity of Liquid & $c_{\mathrm{p}}$ & $\mathrm{m}^{2} \mathrm{~s}^{-2} \mathrm{~K}^{-1}$ & $2.17 \times 10^{3}$ & $2.53 \times 10^{3}$ & $4.18 \times 10^{3}$ \\
\hline Thermal Conductivity of Liquid & $k$ & $\mathrm{~kg} \mathrm{~ms}^{-3} \mathrm{~K}^{-1}$ & 0.161 & 0.203 & 0.604 \\
\hline Surface Tension of Liquid & $\sigma$ & $\mathrm{kg} \mathrm{s}^{-2}$ & $2.38 \times 10^{-2}$ & $2.23 \times 10^{-2}$ & $7.25 \times 10^{-2}$ \\
\hline Gradient of Surface Tension of Liquid & $-\mathrm{d} \sigma / \mathrm{d} T$ & $\mathrm{~kg} \mathrm{~s}^{-2} \mathrm{~K}^{-1}$ & $1.12 \times 10^{-4}$ & $7.73 \times 10^{-5}$ & $1.68 \times 10^{-4}$ \\
\hline Saturation Concentration of Vapour & $c_{\text {sat }}$ & $\mathrm{kg} \mathrm{m}^{-3}$ & 0.637 & 0.186 & $1.94 \times 10^{-2}$ \\
\hline Gradient of Saturation Concentration of Vapour & $\mathrm{d} c_{\mathrm{sat}} / \mathrm{d} T$ & $\mathrm{~kg} \mathrm{~m}^{-3} \mathrm{~K}^{-1}$ & $2.84 \times 10^{-2}$ & $9.47 \times 10^{-3}$ & $1.11 \times 10^{-3}$ \\
\hline Coefficient of Diffusion of Vapour in Air & $D$ & $\mathrm{~m}^{2} \mathrm{~s}^{-1}$ & $1.06 \times 10^{-5}$ & $1.50 \times 10^{-5}$ & $2.44 \times 10^{-5}$ \\
\hline
\end{tabular}

TABLE 3. Physical properties of the three liquids used at temperature $T_{\mathrm{a}}=295 \mathrm{~K}$ and pressure $p_{\mathrm{a}}=99.8 \mathrm{kPa}$. The values of $M$ are given by Tennent (1971), the values of $\rho, \mathscr{L}, \mu, k, \sigma$ and $-\mathrm{d} \sigma / \mathrm{d} T$ were calculated from empirical expressions given by Lide $\&$ Kehiaian (1994), the values of $c_{\mathrm{p}}$ were interpolated from the data given by Raznjevic (1995) (acetone and water) and Tanaka, Fujita \& Uematsu (2007) (methanol), the values of $c_{\text {sat }}$ and $\mathrm{d} c_{\mathrm{sat}} / \mathrm{d} T$ were interpolated from the data given by Reid, Prausnitz \& Poling (1987) (acetone), Perry \& Green (1997) (methanol) and Raznjevic (1995) (water), and the values of $D$ were extrapolated from the data given by Lugg (1968) (acetone and methanol) and were calculated from an empirical expression given by Monteith (1973) (water).

thin layer of $\mathrm{Al}$ with thickness in the range $0.5-1 \mu \mathrm{m}$ using a sputtering process. $\dagger$ The surface roughness of the substrates was measured using a ZYGO profilometer (a microscope interferometer from ZYGO Corporation), and were found to have roughnesses of the same order, namely $306 \mathrm{~nm}$ for aluminium, 440 $\mathrm{nm}$ for titanium, $276 \mathrm{~nm}$ for Macor and $240 \mathrm{~nm}$ for PTFE.

Droplets of three different liquids were used, namely acetone, methanol and ultrapure deionised water, the latter produced using a Milli-Q ultrapure water-purification system from Millipore. Relevant physical

$\dagger$ Note that although we have not directly measured the thickness of the layer of Al, we believe that the range of values given here is more accurate than the figure of $3 \mu \mathrm{m}$ previously stated by David, Sefiane \& Tadrist (2007) and Dunn et al. (2008). 


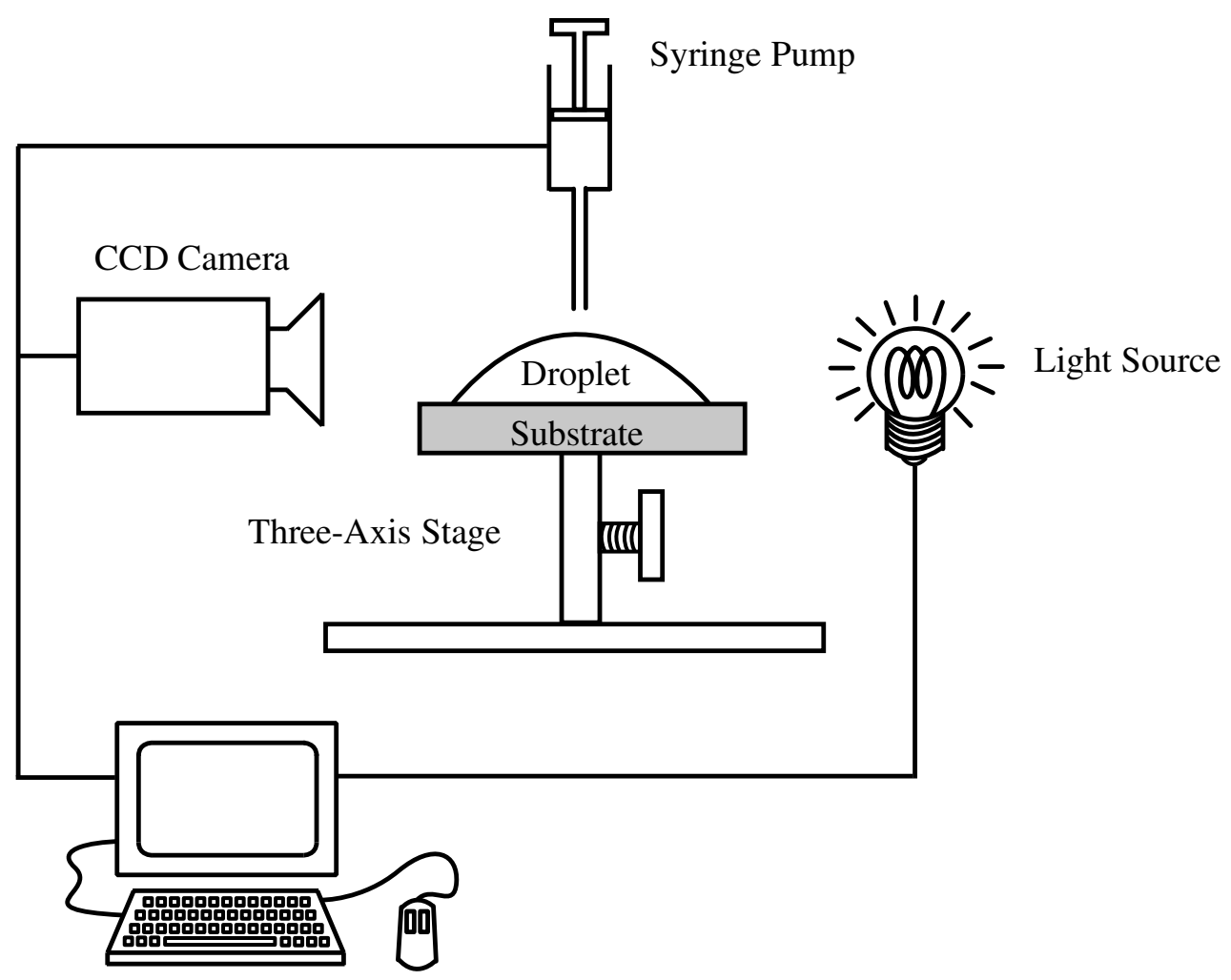

Computer with DSA Software

FIGURE 1. Schematic diagram illustrating the experimental set-up, including the droplet, the substrate, the manually controlled three-axis stage, the charge-coupled device (CCD) camera, the light source, the computer controlled syringe pump, and the computer with Droplet Shape Analysis (DSA) software.

properties of the liquids used are listed in Table 3. Typically droplets had a volume in the range $0.5-8 \mu \mathrm{l}$ and a base radius in the range $0.7-1.8 \mathrm{~mm}$. Typical initial contact angles observed experimentally are $40^{\circ}$ for acetone, $43^{\circ}$ for methanol, and $60^{\circ}$ for water.

All of the experiments were carried out in an atmosphere of air whose temperature was kept at $T_{\mathrm{a}}=$ $295 \mathrm{~K}$ (with a precision of $\pm 1 \mathrm{~K}$ ) using an air-conditioning unit. The pressure of the atmosphere, $p_{\mathrm{a}}$, was monitored to ensure that all of the experiments were performed at the same pressure, namely $p_{\mathrm{a}}=99.8$ $\mathrm{kPa}$. The relative saturation (or, in the case of water, the relative humidity) of the atmosphere is defined to be the ratio of the concentration of vapour in the atmosphere far from the droplet to the saturation concentration. For droplets of water the relative humidity of the atmosphere was also monitored to ensure 
that all of the experiments were performed at the same relative humidity, namely $H=0.4$. For droplets of acetone and methanol the relative saturation is always zero, i.e. $H=0$. For the range of sizes of droplets considered, the droplet shape was always found to be approximately a spherical cap. The droplet profile (specifically its contact angle, base diameter and volume) was measured optically using a DSA 100 Droplet Shape Analysis system from KRÜSS, and hence the total evaporation rate calculated. All of the measurements were performed at least three times to verify the reproducibility of the results, and the optical measurements were calibrated by making direct measurements of the droplet volume using a GR-202 analytical balance from AND with a precision of $\pm 0.02 \mathrm{mg} . \dagger$ The temperature within the droplet was measured to an accuracy of $0.1 \mathrm{~K}$ using a miniature thermocouple which could be placed at various positions within the droplet by raising or lowering the substrate using the manually controlled three-axis stage shown in Figure 1. Care was taken to ensure that the temperature of the thermocouple reached a quasi-static state before each measurement was taken. As David, Sefiane \& Tadrist (2007)[Figure 6] shows, the introduction of the thermocouple does not have a significant effect on the shape of the droplet on the scale of the entire droplet. Further details of the experimental procedure are given by David (2007).

Various other physical effects, including the influence of using different ambient gases and of varying the atmospheric pressure, of using droplets of binary mixtures rather than pure liquids, and of using structured substrates, were also investigated experimentally, but are not considered in the present work (see David (2007) for details).

\section{Mathematical Model}

Motivated by the results of the experiments described in the previous section, we present a mathematical model representing the quasi-steady diffusion-limited evaporation of an axisymmetric sessile droplet of incompressible Newtonian liquid with constant density $\rho$, surface tension $\sigma$, specific heat capacity $c_{\mathrm{p}}$ and

$\dagger$ The quoted value of the precision is that given by the manufacturer. However, we believe that this figure is somewhat conservative and that the value of $\pm 0.01 \mathrm{mg}$ given by David, Sefiane \& Tadrist (2007) is probably more realistic. 


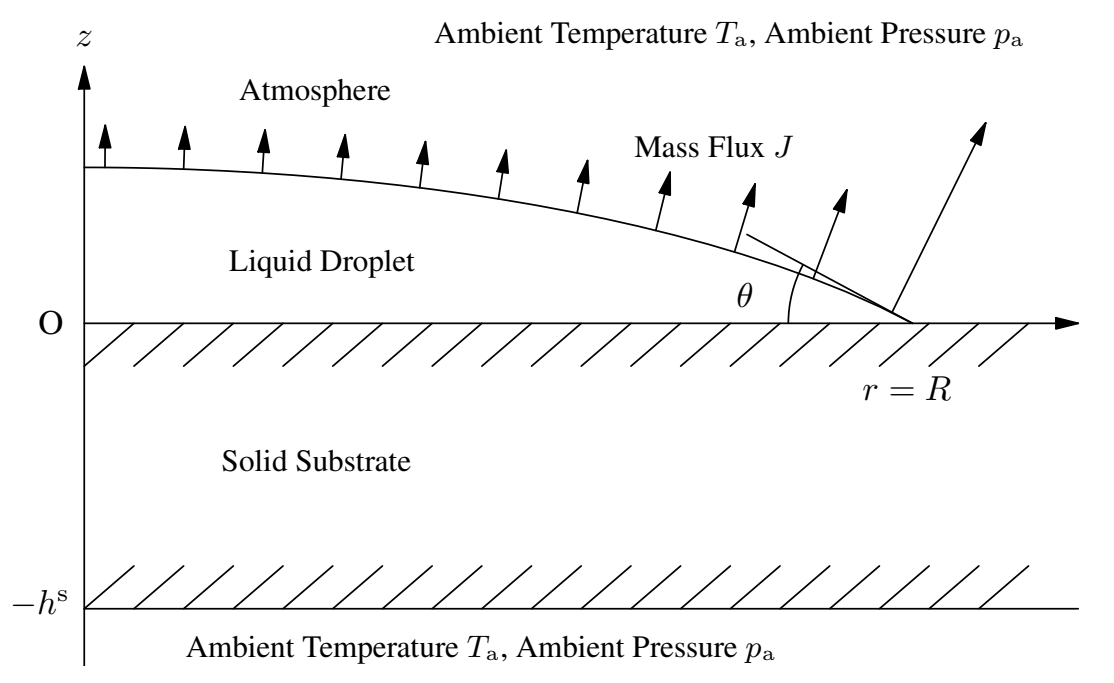

FIGURE 2. The geometry of the problem.

thermal conductivity $k$ on a horizontal substrate of constant thickness $h^{\mathrm{s}}$ with constant density $\rho^{\mathrm{s}}$, specific heat capacity $c_{\mathrm{p}}^{\mathrm{s}}$ and thermal conductivity $k^{\mathrm{s}}$. Referred to cylindrical polar coordinates $(r, \phi, z)$ with origin on the substrate at the centre of the droplet and with the $z$ axis vertically upwards, the shape of the free surface of the droplet at time $t$ is denoted by $z=h(r, t)$, the upper surface of the substrate by $z=0$, and the lower surface of the substrate by $z=-h^{\mathrm{s}}$, as shown in Figure 2. Note that, unlike Dunn et al. (2008), we do not assume that either the droplet or the substrate is thin.

In the experiments the contact lines of the droplets are typically pinned by surface-roughness effects over much of the lifetime of the droplet, but typically de-pin prior to complete evaporation. All of the experimental results for temperature and evaporation rate reported by David, Sefiane \& Tadrist (2007) and in the present work are for droplets in the pinned stage of the evaporation process, and so in the present mathematical model we will assume that the contact line of the droplet remains pinned so that the droplet radius, denoted by $R$, remains constant. The atmosphere surrounding the droplet and the substrate is assumed to be at constant atmospheric temperature $T_{\mathrm{a}}$ and pressure $p_{\mathrm{a}}$.

In the experiments the (reduced) Reynolds number $R_{\mathrm{e}}{ }^{*}=\left(h_{\mathrm{m}}(0) / R\right)^{2} R_{\mathrm{e}}$, where $h_{\mathrm{m}}=h_{\mathrm{m}}(t)=h(0, t)$ is the maximum height of the droplet and $R_{\mathrm{e}}=\rho U R / \mu$ is the usual Reynolds number in which $U$ is a 
characteristic radial velocity, is small, and so inertial effects are negligible. $\dagger$ Moreover, since the maximum height of the droplets is typically less than the capillary length $\ell=(\sigma / \rho g)^{1 / 2}$ (equal to $1.75 \times 10^{-3} \mathrm{~m}$, $1.70 \times 10^{-3} \mathrm{~m}$ and $2.72 \times 10^{-3} \mathrm{~m}$ for acetone, methanol and water, respectively), where $g$ denotes the magnitude of acceleration due to gravity, we assume that surface-tension effects dominate gravitational effects and so the free surface of the droplet is a spherical cap given by

$$
h(r, t)=\sqrt{\left(\frac{R}{\sin \theta}\right)^{2}-r^{2}}-\frac{R}{\tan \theta}
$$

with contact angle $\theta=\theta(t)$ and volume $V=V(t)$ given by

$$
V=\frac{\pi h_{\mathrm{m}}\left(3 R^{2}+h_{\mathrm{m}}^{2}\right)}{6}
$$

where $h_{\mathrm{m}}=R \tan (\theta / 2)$. The total evaporation rate is given by

$$
-\frac{\mathrm{d} V}{\mathrm{~d} t}=\frac{2 \pi}{\rho} \int_{0}^{R} J \sqrt{1+\left(\frac{\partial h}{\partial r}\right)^{2}} r \mathrm{~d} r
$$

where $J=J(r, t)(\geq 0)$ is the local evaporative mass flux from the droplet.

In the experiments the (reduced) Péclet number $P_{\mathrm{e}}{ }^{*}=\left(h_{\mathrm{m}}(0) / R\right)^{2} P_{\mathrm{e}}$, where $P_{\mathrm{e}}=\rho c_{\mathrm{p}} U R / k$ is the usual Péclet number, is small, and so thermal convection is negligible. Moreover, since the characteristic timescale for thermal conduction in the droplet $\rho c_{\mathrm{p}} h_{\mathrm{m}}(0)^{2} / k$ (equal to 2.6, 2.8 and $4.2 \mathrm{~s}$ for droplets of radius $R=1.35 \mathrm{~mm}$ of acetone, methanol and water, respectively) is much less than the lifetime of the droplets in the experiments (typically of the order of 35,80 and $700 \mathrm{~s}$ for acetone, methanol and water, respectively), we assume that thermal conduction in the liquid is quasi-steady and hence the temperature of the liquid, denoted by $T=T(r, z, t)$, satisfies Laplace's equation

$$
\nabla^{2} T=0
$$

Similarly, since the characteristic timescale for thermal conduction in the substrate $\rho^{\mathrm{s}} c_{\mathrm{p}}^{\mathrm{s}} h^{\mathrm{s} 2} / k^{\mathrm{s}}$ (equal to $\dagger$ A more careful analysis like that of Ristenpart et al. (2007) shows that this conclusion holds provided that any singularity in the local mass flux at the contact line is integrable. A similar argument justifies the neglect of thermal convection in the temperature equation. 
approximately $0.01,0.1,1$ and $10 \mathrm{~s}$ for $\mathrm{Al}, \mathrm{Ti}$, Macor and PTFE, respectively) is much less than the lifetime of the droplets in the experiments, we assume that thermal conduction in the substrate is quasi-steady and hence the temperature of the substrate, denoted by $T^{\mathrm{s}}=T^{\mathrm{s}}(r, z, t)$, also satisfies Laplace's equation

$$
\nabla^{2} T^{\mathrm{s}}=0
$$

The mass flux from the droplet satisfies the local energy balance

$$
\mathscr{L} J=-k \nabla T \cdot \mathbf{n}
$$

on $z=h$ for $r<R$, where $\mathscr{L}$ is the latent heat of vaporisation and $\mathbf{n}=\left(-h_{r}, 0,1\right) / \sqrt{1+h_{r}^{2}}$ is the unit outward normal to the free surface of the droplet. We assume that both the temperature and the heat flux are continuous between the droplet and the wetted surface of the substrate, so that

$$
T=T^{\mathrm{s}}, \quad-k \frac{\partial T}{\partial z}=-k^{\mathrm{s}} \frac{\partial T^{\mathrm{s}}}{\partial z}
$$

on $z=0$ for $r<R$, and that the temperature of the unwetted surface of the substrate is equal to the atmospheric value, i.e.

$$
T^{\mathrm{s}}=T_{\mathrm{a}}
$$

on $z=0$ for $r>R$ and on $z=-h^{\mathrm{s}}$.

Assuming that the atmosphere is quiescent (we shall revisit this assumption in section 6.2), vapour transport in the atmosphere is solely by diffusion. Moreover, since the characteristic timescale for diffusion $R^{2} / D$, where $D$ is the coefficient of diffusion of vapour in the atmosphere (equal to $0.17,0.12$ and $0.07 \mathrm{~s}$ for droplets of radius $R=1.35 \mathrm{~mm}$ of acetone, methanol and water, respectively), is much less than the lifetime of the droplets in the experiments, we assume that the diffusion of vapour in the atmosphere is quasi-steady and hence the concentration of vapour in the atmosphere above the droplet and the substrate (i.e. the mass of vapour per unit volume of atmosphere), denoted by $c=c(r, z, t)$, also satisfies Laplace's 
equation

$$
\nabla^{2} c=0
$$

We assume that the atmosphere just above the free surface of the droplet is saturated with vapour, so that $c=c_{\mathrm{sat}}(T)$ on $z=h$ for $r<R$ (see, for example, Popov (2005)), where the saturation concentration $c_{\mathrm{sat}}=c_{\mathrm{sat}}(T)$ is assumed to be a linearly increasing function of temperature given by

$$
c_{\mathrm{sat}}(T)=c_{\mathrm{sat}}\left(T_{\mathrm{a}}\right)+\left.\frac{\mathrm{d} c_{\mathrm{sat}}}{\mathrm{d} T}\right|_{T=T_{\mathrm{a}}}\left(T-T_{\mathrm{a}}\right)
$$

(see, for example, Sultan, Boudaoud \& Ben Amar (2005)). Note that the linear approximation (3.10) is appropriate for the relatively small evaporative cooling of a few degrees in the present experiments, but will, of course, fail in situations (such as, for example, experiments with significantly reduced atmospheric pressures) with larger evaporative cooling. On the unwetted surface of the substrate there is no mass flux, i.e.

$$
\frac{\partial c}{\partial z}=0
$$

on $z=0$ for $r>R$, and far from the droplet the concentration of vapour approaches its ambient value, i.e.

$$
c \rightarrow H c_{\text {sat }}\left(T_{\mathrm{a}}\right)
$$

as $\left(r^{2}+z^{2}\right)^{1 / 2} \rightarrow \infty$, where again $H$ is the relative saturation far from the droplet. Once $c$ is known the local mass flux from the free surface of the droplet is given by

$$
J=-D \nabla c \cdot \mathbf{n}
$$

on $z=h$ for $r<R$, where again $D$ is the coefficient of diffusion of vapour in the atmosphere.

When the variation of the saturation concentration with temperature is negligible (i.e. when $c_{\text {sat }}(T) \simeq$ $\left.c_{\mathrm{sat}}\left(T_{\mathrm{a}}\right)\right)$, so that the concentration of vapour at the free surface of the droplet is constant, the present model reduces to the basic model described in section 1 for which the problem for the concentration of 
vapour in the atmosphere decouples from the problem for the temperature in the liquid and the substrate. In this case the exact solution for the concentration is well known (see, for example, Poulard, Guéna \& Cazabat (2005) and Popov (2005)), the total evaporation rate is independent of the thermal conductivity of the substrate, and the temperature of the droplet and the substrate can be calculated a posteriori if required. For a thin droplet (specifically, at leading order in the limit $\theta \rightarrow 0$ ) the total evaporation rate is given by

$$
-\frac{\mathrm{d} V}{\mathrm{~d} t}=\frac{4 R D(1-H) c_{\mathrm{sat}}\left(T_{\mathrm{a}}\right)}{\rho}
$$

in the special case $\theta=\pi / 2$ it is given by

$$
-\frac{\mathrm{d} V}{\mathrm{~d} t}=\frac{2 \pi R D(1-H) c_{\mathrm{sat}}\left(T_{\mathrm{a}}\right)}{\rho}
$$

while for intermediate values of $\theta \mathrm{Hu} \&$ Larson (2002) used a finite-element method to obtain a simple approximate expression for the total evaporation rate, namely

$$
-\frac{\mathrm{d} V}{\mathrm{~d} t}=\frac{\pi R D(1-H) c_{\mathrm{sat}}\left(T_{\mathrm{a}}\right)}{\rho}\left(0.27 \theta^{2}+1.30\right)
$$

The present model generalises and improves the basic model by including the variation of the saturation concentration with temperature as proposed by Sultan, Boudaoud \& Ben Amar (2005), and hence coupling the problems for the vapour concentration in the atmosphere and the temperature in the liquid and the substrate. In particular, the total evaporation rate predicted by the present model will depend on the thermal conductivity of the substrate.

\section{Numerical Procedure}

In general, the coupled problem for $T, T^{\mathrm{s}}$ and $c$ has to be solved numerically, and this was done using a finite-element method implemented using the MATLAB-based numerical analysis package COMSOL Multiphysics (formerly FEMLAB, see COMSOL (2007)). The far-field condition was imposed on a notional computational boundary at a distance $320 R$ from the origin. The initial mesh, comprising triangular elements, was restricted so that on the free surface $\underset{14}{z}=h$ the vertices of the elements were a maximum 
of $R / 200$ apart with a maximum growth rate of $10 \%$. In addition, in order to improve the accuracy of the solution near the contact line $r=R$, a semi-circular sub-domain of radius $R / 10$ with a maximum element size of $R / 100$ was used near $r=R$. The overall mesh was then refined by dividing each element into four smaller triangular elements to produce a final mesh with approximately 60,000 elements. The non-linear stationary PDE solver "femnlin" was then used to solve the system. The procedure (a generalisation of that used by Dunn et al. (2008)) was verified by recovering the known analytical solution (3.15) for the total evaporation rate given by the basic model in the case $\theta=\pi / 2$ to an accuracy of $0.3 \%$, and by confirming that the results are insensitive to further grid refinement. Note that decreasing the notional computational boundary to a distance of only $160 R$ from the origin increased the error to $0.6 \%$. Furthermore, note that Hu \& Larson (2002) used a notional computational boundary a distance of only $20 R$ from the origin, and our computations suggests that this may introduce an error of approximately $5 \%$ in their results.

Having determined the quasi-static solution for $c$ for a particular droplet volume (i.e. for a particular contact angle) a simple application of Euler's forward method was used to calculate the new value of the droplet volume (i.e. the new value of the contact angle) and the process marched forward in time until the contact angle reached the value at which the droplet typically de-pinned in the experiments, denoted by $\theta_{\mathrm{d}}$ (equal to $30^{\circ}$ for acetone and methanol and $20^{\circ}$ for water), at which point the computations were terminated.

\section{Results}

Figure 3 shows a comparison between the experimentally measured and theoretically predicted values of the droplet volume $V$ for a droplet of methanol of radius $R=1.44 \mathrm{~mm}$ on substrates of $\mathrm{Al}$ and PTFE as a function of time $t$. The error bars on the experimental results in Figure 3 are $\pm 0.025 \mu \mathrm{l}$ and correspond to the precision of the analytical balance of $\pm 0.02 \mathrm{mg}$. Note that the experimental results are shown only until the variation of $V$ with $t$ begins to vary significantly from linear behaviour. As Figure 3 illustrates, 


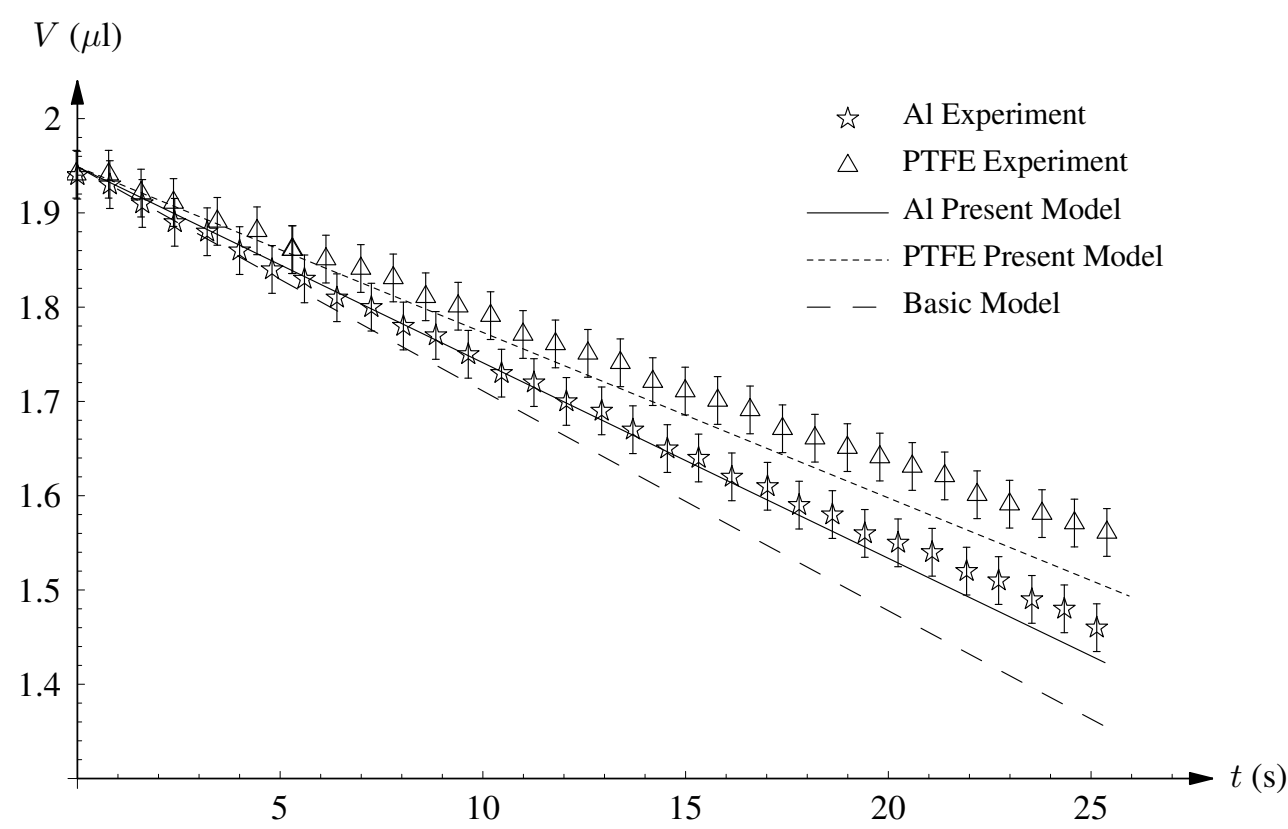

FIGURE 3. Comparison between the experimentally measured and theoretically predicted values of the droplet volume $V$ for a droplet of methanol of radius $R=1.44 \mathrm{~mm}$ on substrates of $\mathrm{Al}$ and PTFE as a function of time $t$. The error bars on the experimental results are $\pm 0.025 \mu \mathrm{l}$ and correspond to the precision of the analytical balance of $\pm 0.02 \mathrm{mg}$.

typically the theoretical predictions accurately capture the very nearly linear variation of $V$ with $t$. For reference, Figure 3 also includes the theoretical prediction of the basic model (which does not depend on the thermal conductivity of the substrate and hence somewhat over-predicts the evaporation rate). Note that the corresponding experimentally measured and theoretically predicted values of the contact angle (not shown for brevity) are also monotonically decreasing functions of $t$ (see, for example, the experimentally measured values reported by David, Sefiane \& Tadrist (2007)[Figure 4]). In what follows we compare values of the average total evaporation rate defined by

$$
-\frac{1}{\theta_{0}-\theta_{\mathrm{d}}} \int_{\theta_{\mathrm{d}}}^{\theta_{0}} \frac{\mathrm{d} V}{\mathrm{~d} t} \mathrm{~d} \theta
$$

where $\theta_{0}=\theta(0)$, as a function of droplet radius $R$.

Figure 4 shows a bar chart summarising the theoretically predicted values of the total evaporation rate for droplets of acetone, methanol and water of radius $R=1.35 \mathrm{~mm}$ on substrates of $\mathrm{Al}$, Ti, Macor and 


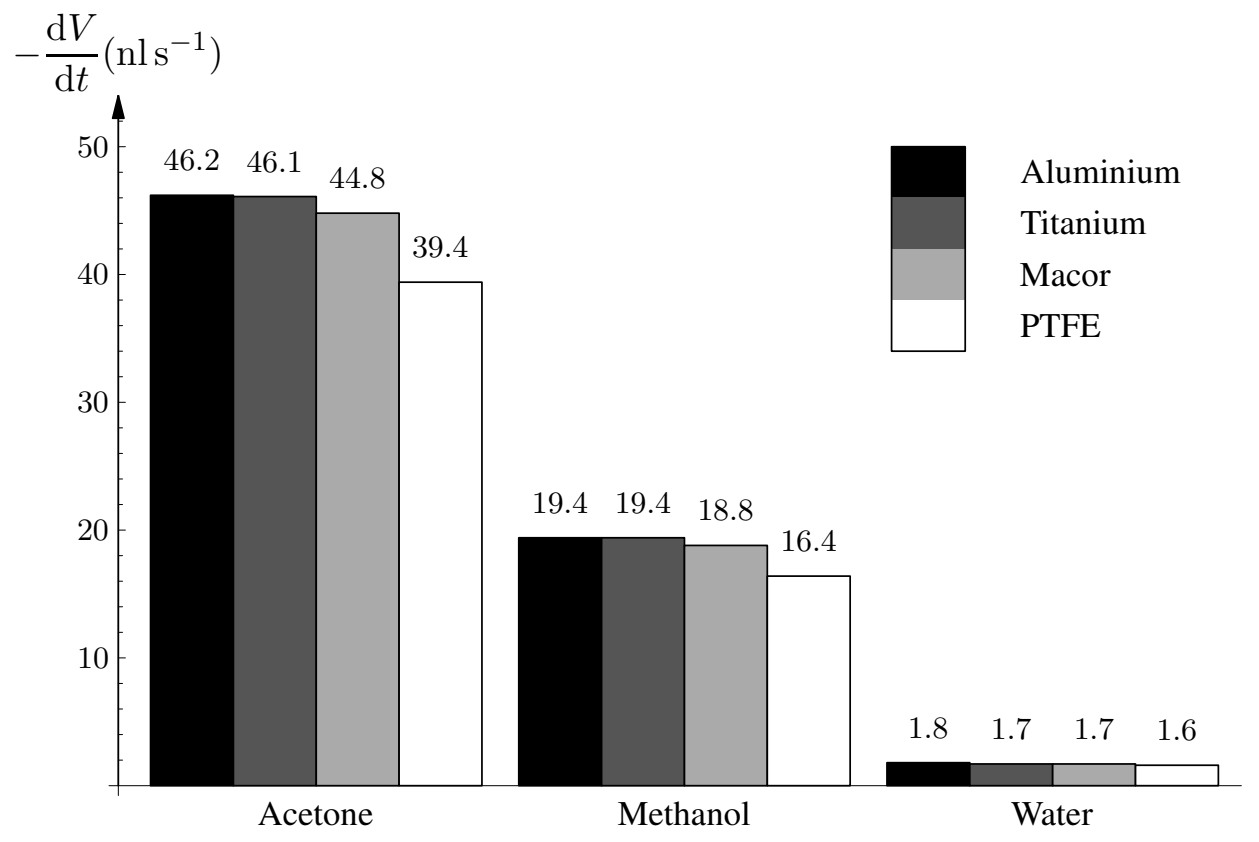

FIGURE 4. Bar chart summarising the theoretically predicted values of the total evaporation rate for droplets of acetone, methanol and water of radius $R=1.35 \mathrm{~mm}$ on substrates of $\mathrm{Al}$, Ti, Macor and PTFE.

PTFE, and clearly illustrates the strong influence of both the nature of the liquid and the thermal properties of the substrate.

Figures 5 and 6 show a comparison between experimentally measured and theoretically predicted values of the average total evaporation rate plotted as a function of droplet radius $R$ for droplets of acetone, methanol and water on $\mathrm{Al}$ and PTFE substrates. For reference, Figures 5 and 6 also include the theoretical prediction of the basic model (which again does not depend on the thermal conductivity of the substrate and hence somewhat over-predicts the evaporation rate). In particular, Figure 5 shows that (in agreement with the results of previous authors such as, for example, Birdi, Vu \& Winter (1989)) the evaporation rate is approximately a linear function of the droplet radius $R$. Figure 5 shows that the theoretical predictions of the present model for droplets of acetone and methanol are in good qualitative agreement with the experimental results for both substrates (albeit slightly over-predicting the total evaporation rate on a PTFE substrate). However, Figure 6, an enlargement of the lower part of Figure 5 showing the results for droplets of water in more detail, shows that both the present model and the basic model systematically under-predict 


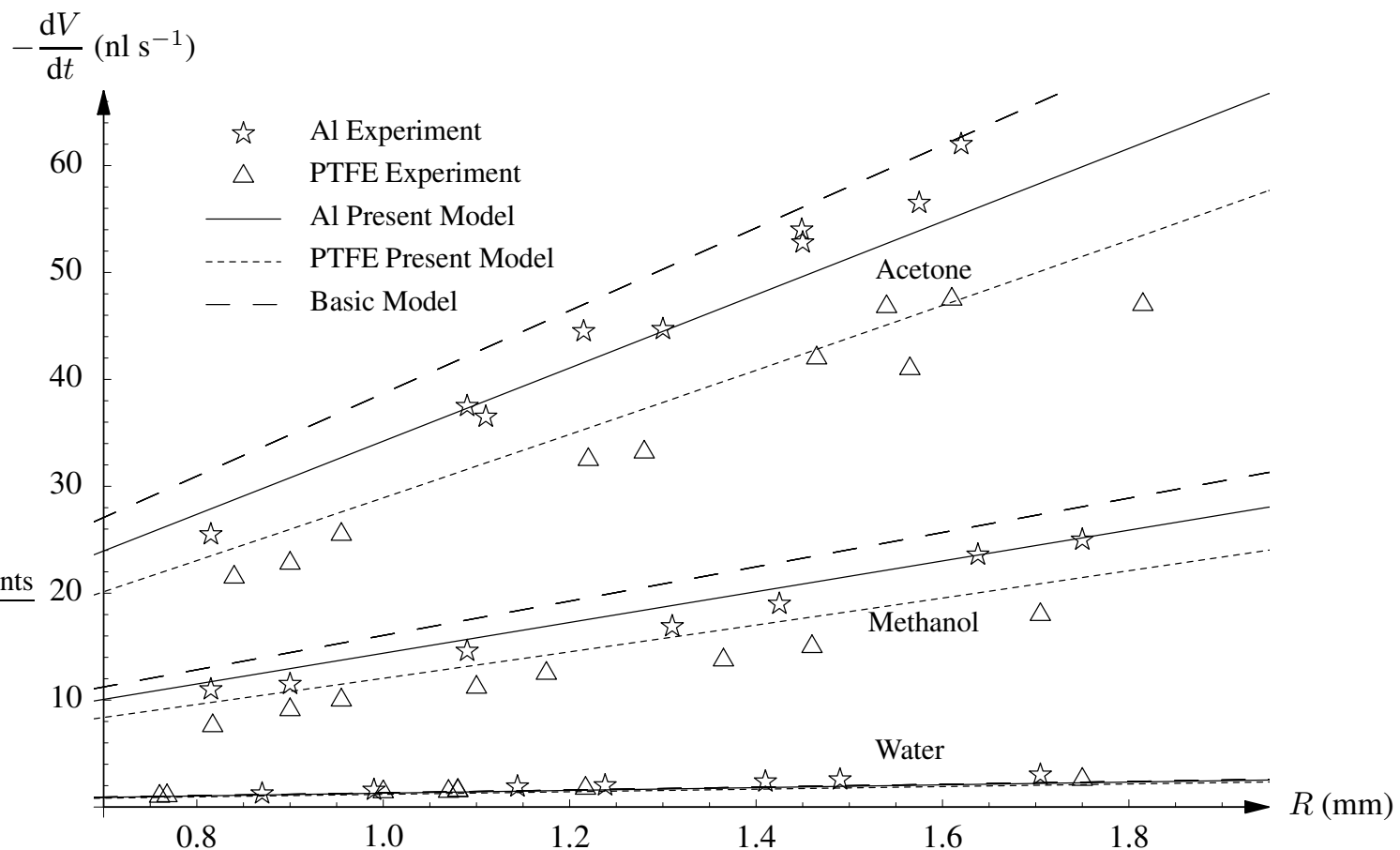

FIGURE 5. Comparison between experimentally measured and theoretically predicted values of the average total evaporation rate plotted as a function of droplet radius $R$ for droplets of acetone, methanol and water on $\mathrm{Al}$ and PTFE substrates.

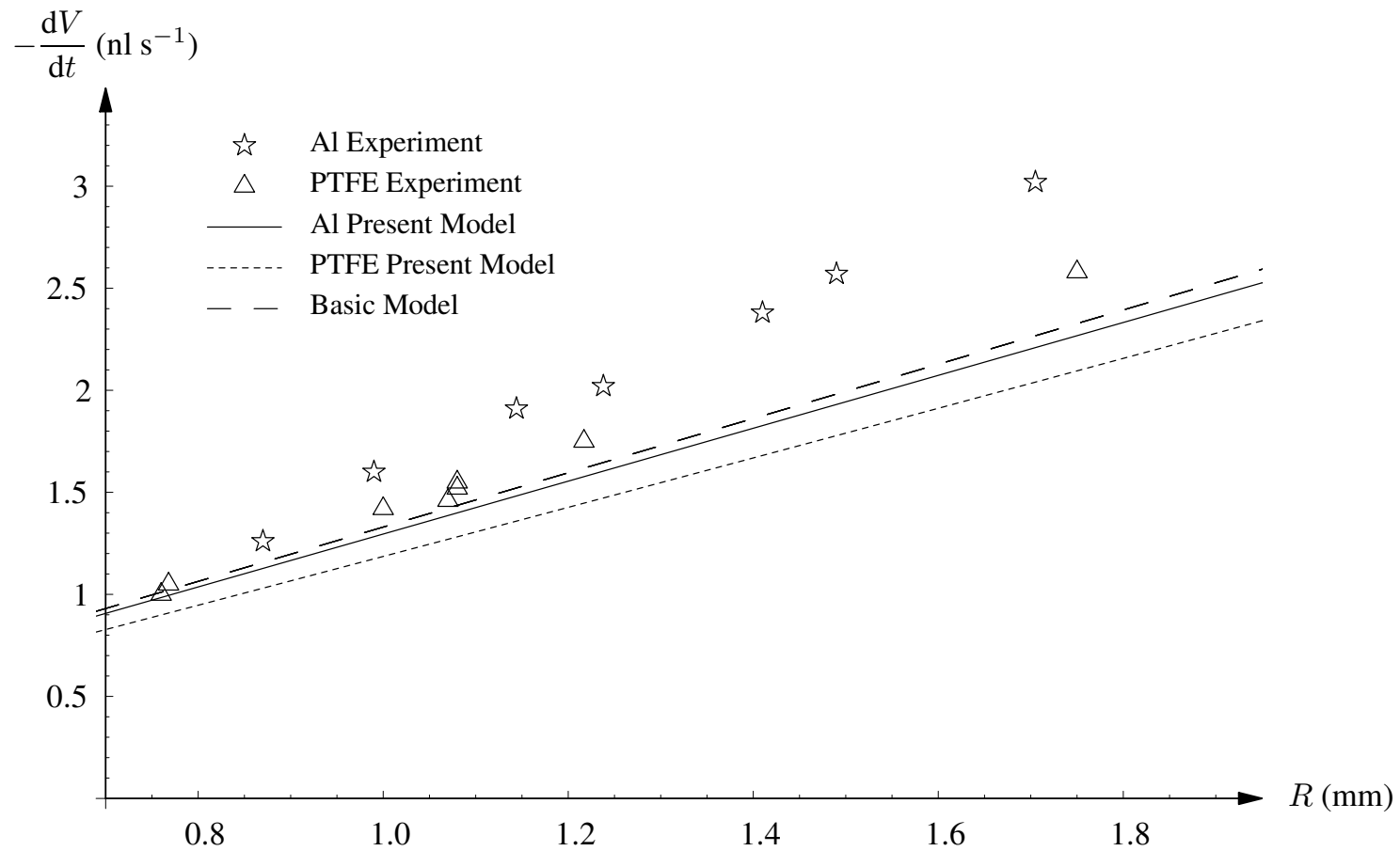

FIGURE 6. An enlargement of the lower part of Figure 5 showing the results for droplets of water in more detail. 


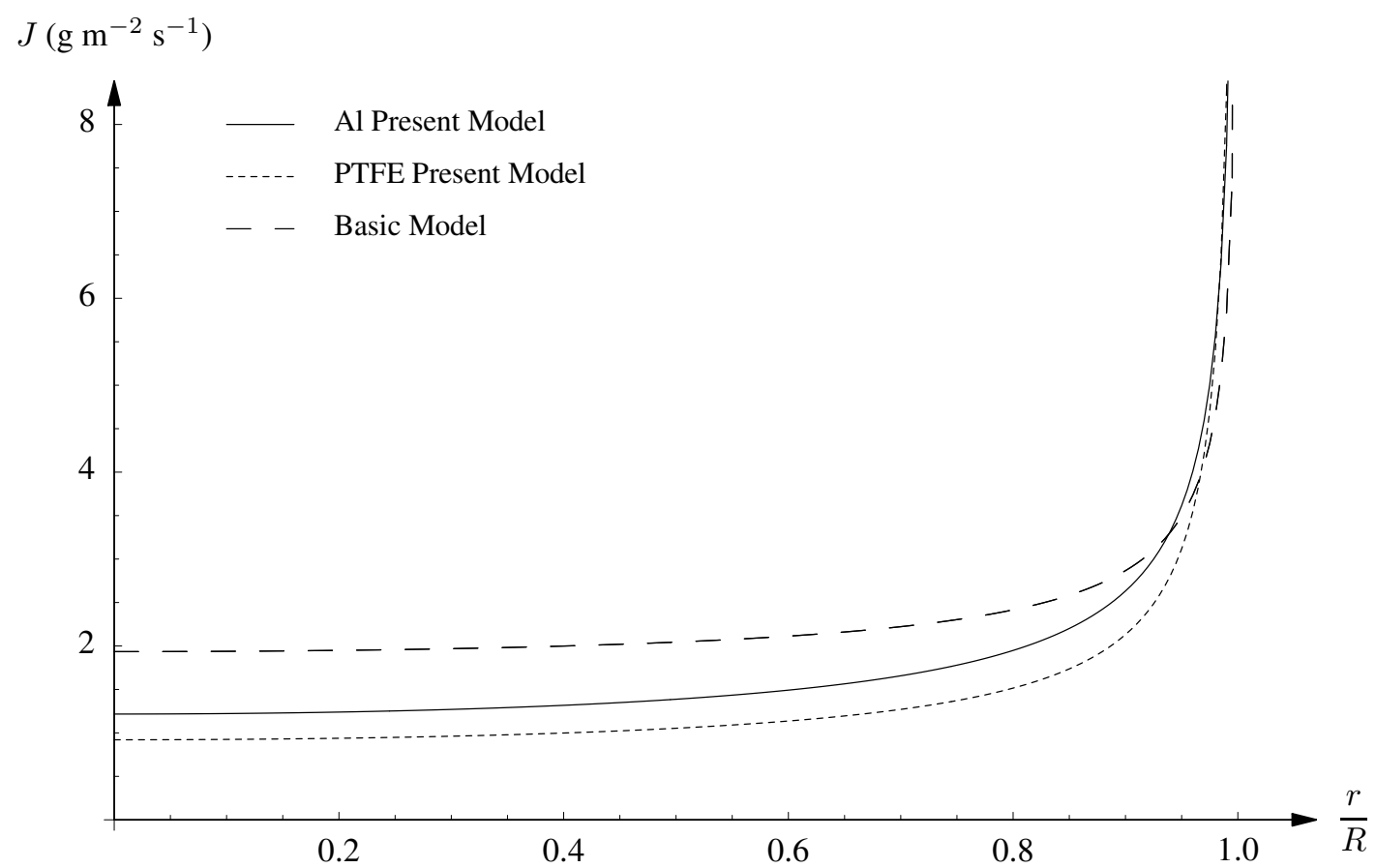

FIGURE 7. Theoretical predictions for the initial evaporative mass flux from droplets of methanol of radius $R=1.35 \mathrm{~mm}$ on $\mathrm{Al}$ and PTFE substrates as a function of $r / R$.

the experimental results for droplets of water; we shall revisit this issue in section 6.2. Nevertheless, in view of the many assumptions made in deriving the present model, the agreement (at least for acetone and methanol) is remarkably good, especially as there are no fitting parameters in the model, and no tuning of the values of the physical parameters has taken place. Nevertheless further refinements to the present model are clearly possible, and two ad hoc improvements will be considered subsequently in section 6 . Perhaps the most satisfying aspect of the agreement shown in Figure 5 is the manner in which the present model reproduces the significant difference in the total evaporation rate between droplets of the same liquid on different substrates. Figures 5 and 6 also show that the theoretical predictions of the basic model are closer to those of the present model for an Al substrate than for a PTFE substrate. This is because $\mathrm{Al}$ is a better conductor than PTFE and hence the evaporative cooling on an Al substrate is much less than that on a PTFE substrate, and hence the saturation concentration of vapour at the free surface is closer to the constant value of $c_{\text {sat }}\left(T_{\mathrm{a}}\right)$ assumed in the basic model. 

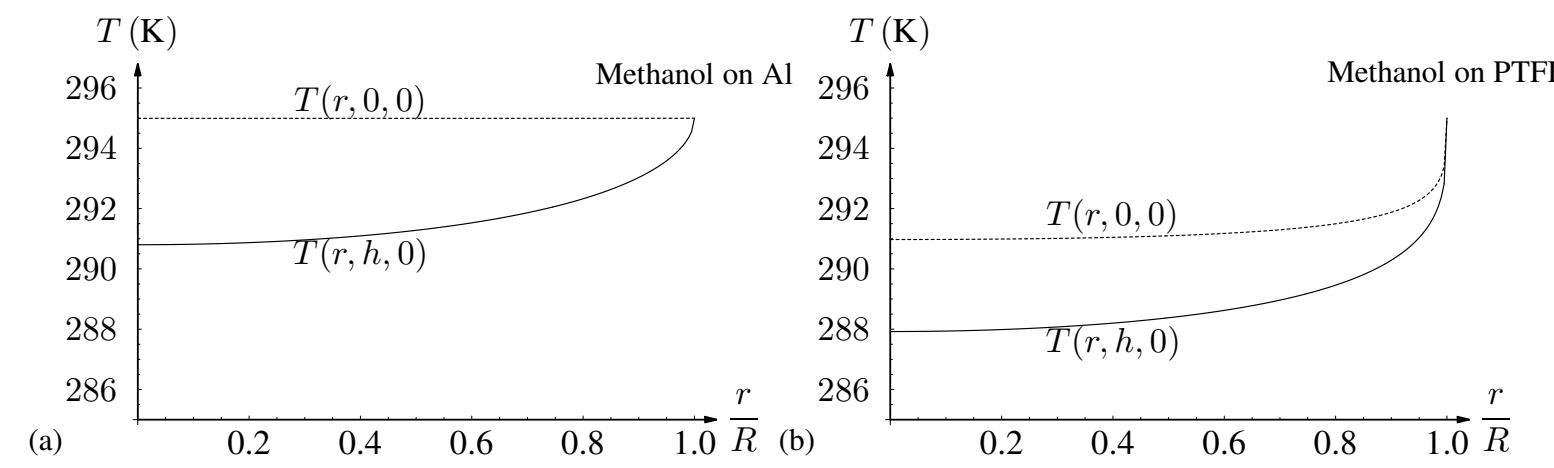

FIGURE 8. Theoretical predictions of the present model for the initial temperatures of the free surface of the droplet, $T(r, h, 0)$, and the wetted surface of the substrate, $T(r, 0,0)$, for a droplet of methanol of radius $R=1.35 \mathrm{~mm}$ on (a) $\mathrm{Al}$ and (b) PTFE substrates as functions of $r / R$.

Figure 7 shows the theoretical predictions for the initial evaporative mass flux from droplets of methanol of radius $R=1.35 \mathrm{~mm}$ on $\mathrm{Al}$ and PTFE substrates as a function of $r / R$. In particular, it shows that the mass flux is singular at the contact line, just as Deegan et al. (1997) found for the basic model and Dunn et al. (2008) found in the special case of a thin droplet on a thin substrate.

Figure 8 shows the theoretical predictions of the present model for the initial temperatures of the free surface of the droplet, $T(r, h, 0)$, and the wetted surface of the substrate, $T(r, 0,0)$, for a droplet of methanol of radius $R=1.35 \mathrm{~mm}$ on (a) $\mathrm{Al}$ and (b) PTFE substrates as functions of $r / R$. Figure 9 shows the theoretical predictions of the present model for the initial temperature contours in the $r, z$-plane within the substrate and a droplet of $(\mathrm{a}, \mathrm{b})$ acetone, $(\mathrm{c}, \mathrm{d})$ methanol and $(\mathrm{e}, \mathrm{f})$ water of radius $R=1.35 \mathrm{~mm}$ on $(\mathrm{a}, \mathrm{c}, \mathrm{e}) \mathrm{Al}$ and $(\mathrm{b}, \mathrm{d}, \mathrm{f})$ PTFE substrates. As expected, the results shown in Figures 8 and 9 confirm that the evaporative cooling means that the free surface is cooler than the wetted surface of the substrate below. The results also show that, since $\mathrm{Al}$ is a relatively good thermal conductor, the temperature of an $\mathrm{Al}$ substrate is almost constant (and equal to the atmospheric temperature $T_{\mathrm{a}}$ ) and essentially all of the evaporative cooling takes place within the droplet (see Figure $9(\mathrm{a}, \mathrm{c}, \mathrm{e})$ ), whereas, since PTFE is a relatively poor thermal conductor, there is significant evaporative cooling within a PTFE substrate (see Figure 9(b,d,f)). However, the results also show that in all the situations investigated the free surface is warmest near the contact line and coolest 


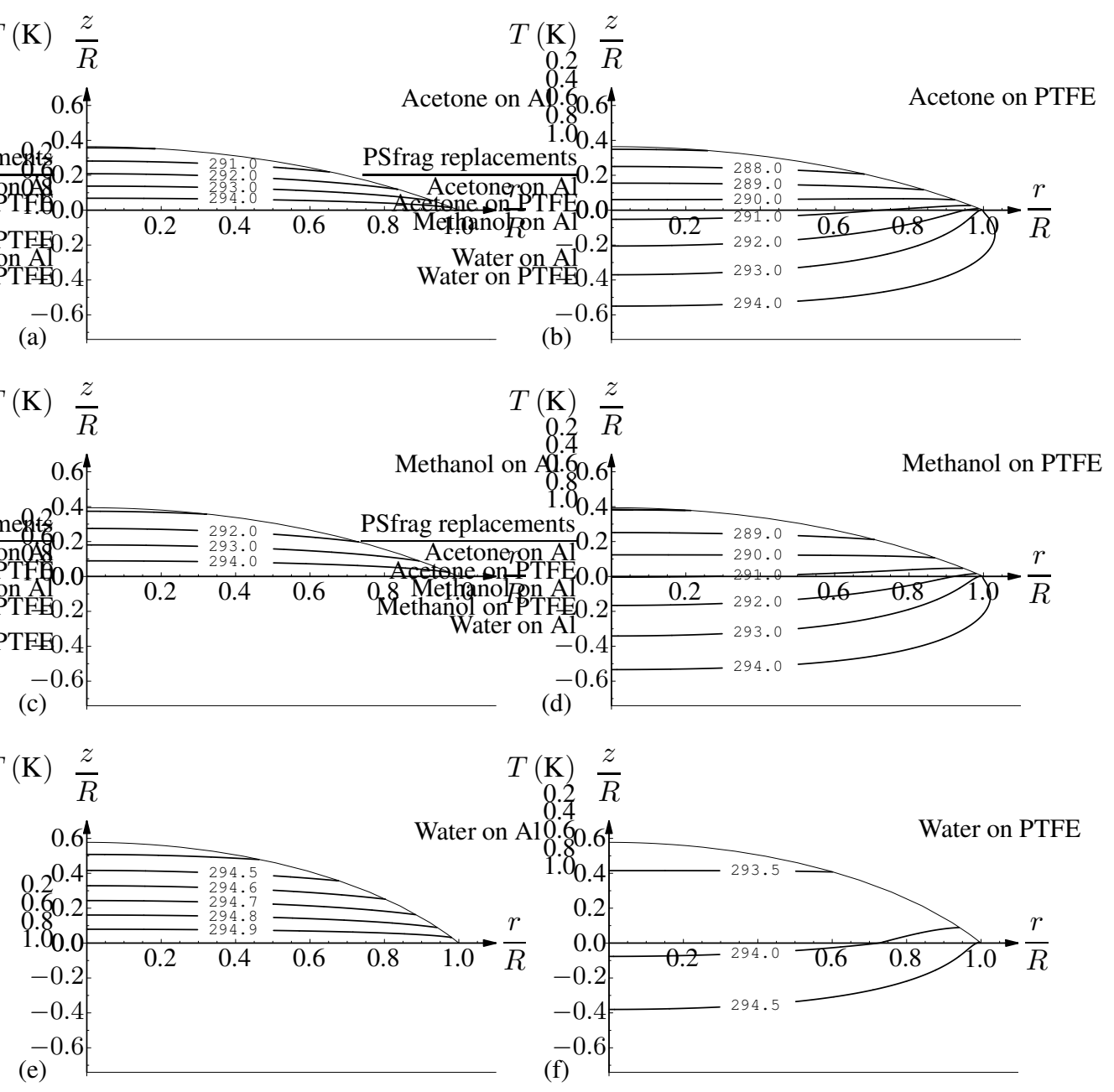

FIGURE 9. Theoretical predictions of the present model for the initial temperature contours in the $r, z$-plane within the substrate and a droplet of (a,b) acetone, (c,d) methanol and (e,f) water of radius $R=1.35 \mathrm{~mm}$ on (a,c,e) Al and (b,d,f) PTFE substrates. at the centre of the droplet. This agrees with the numerical results of Hu \& Larson (2005b)[Figure 2] and the direct experimental measurements of the temperature by David, Sefiane \& Tadrist (2007)[Table 1], but is qualitatively different from the corresponding result given by Dunn et al. (2008)[Figure 2], calculated using a simplified version of the present model in which both the droplet and the substrate were assumed to be thin, which predicts that the droplet is coolest near the contact line (where the mass flux is largest) and warmest at the centre of the droplet (where the mass flux is smallest). The explanation for the present result is simply that in all the situations considered, radial diffusion of heat from $r>R$ (neglected by Dunn et al. (2008)) is strong enough to overcome the effects of evaporative cooling near the contact line 
so that this region is in fact warmer than the centre of the droplet. However, it should be noted that the numerical results of $\mathrm{Hu} \&$ Larson (2005b)[Figure 2] are qualitatively consistent with those of Dunn et al. (2008)[Figure 2] for sufficiently small values of the contact angle, lending support to the simplified model proposed by Dunn et al. (2008) in that situation. In general such a temperature gradient along the free surface will generate a thermocapillary-driven flow from the contact line towards the centre of the droplet which might be strong enough to create a recirculation flow of the kind described by Hu \& Larson (2005b), Xu \& Luo (2007) and Ristenpart et al. (2007). However, as Dunn et al. (2008) discussed, for the present experiments this effect is likely to be weak and so, in the light of the good agreement between the present experimentally measured and theoretically predicted values, will not be considered any further here.

Table 4 shows a comparison between the experimentally measured and theoretically predicted values of the difference between the atmospheric temperature and the average initial temperature in the bulk of a droplet of radius $R=1.35 \mathrm{~mm}$ of all three liquids on all four substrates. In Table 4 the theoretical value of the average initial temperature in the bulk is defined to be

$$
\frac{1}{h_{\mathrm{m}}} \int_{0}^{h_{\mathrm{m}}} T(0, z, 0) \mathrm{d} z
$$

moreover, "Improved Model 1" and "Improved Model 2" refer to two improved versions of the present model discussed subsequently in section 6 . The experimental measurement of the bulk temperature is very sensitive to the precise location of the thermocouple (positioned manually in the center of the droplet) and so the experimental results given in Table 4 should be treated with some caution. In particular, they are not necessarily reproducible to the quoted accuracy of $0.1 \mathrm{~K}$; nevertheless they provide valuable qualitative information about the temperature distributions within the droplets. Note that David, Sefiane \& Tadrist (2007)[Table 1] give an example of temperature measurements taken at several locations within a droplet and the surrounding atmosphere. The predictions of the present theory shown in Table 4 are in reasonable agreement with the experimental results, but consistently under-predict the cooling of droplets of acetone 


$\begin{array}{cccccc}\text { Liquid } & \text { Method } & \text { Aluminium } & \text { Titanium } & \text { Macor } & \text { PTFE } \\ \text { Acetone } & \text { Experiment } & 4.0 & \text { N/A } & \text { N/A } & 8.6 \\ & \text { Basic Model } & 3.4 & 3.5 & 4.7 & 11.0 \\ & \text { Present Model } & 2.2 & 2.3 & 3.1 & 6.0 \\ & \text { Improved Model 1 } & 2.3 & 2.5 & 3.7 & 7.2 \\ \text { Methanol } & \text { Experiment } & 3.6 & \text { N/A } & \text { N/A } & 6.9 \\ & \text { Basic Model } & 2.6 & 2.7 & 3.8 & 9.3 \\ & \text { Present Model } & 1.8 & 1.9 & 2.6 & 5.3 \\ & \text { Improved Model 1 } & 1.8 & 2.0 & 3.2 & 6.4 \\ \text { Water } & \text { Experiment } & 0.0 & 0.0 & 0.6 & 1.3 \\ & \text { Basic Model } & 0.2 & 0.2 & 0.5 & 1.5 \\ & \text { Present Model } & 0.2 & 0.2 & 0.6 & 1.6 \\ & \text { Improved Model 1 } & 0.2 & 0.3 & 0.6 & 1.6 \\ \text { Improved Model 2 } & 0.1 & 0.2 & 0.5 & 1.6\end{array}$

TABLE 4. Comparison between the experimentally measured and theoretically predicted values of the difference (in K) between the atmospheric temperature and the average initial temperature in the bulk of a droplet of radius $R=1.35 \mathrm{~mm}$ of all three liquids on all four substrates. "Improved Model 1" and "Improved Model 2" refer to the improved versions of the present model including Newton's law of cooling (discussed subsequently in section 6.1), and Newton's law of cooling and buoyancy of water vapour in the atmosphere (discussed subsequently in section 6.2), respectively.

and methanol. The predictions for the cooling of a droplet of water is within the error of the experimental measurements.

Figure 10 shows the theoretical predictions of the present model for the contours of the initial concentration of vapour in the atmosphere for a droplet of methanol of radius $R=1.35 \mathrm{~mm}$ on $\mathrm{Al}$ and PTFE substrates, respectively. In particular, Figure 10 clearly illustrates that the concentration of vapour just above the free surface of the droplet is not, in general, constant, as assumed in the basic model. 

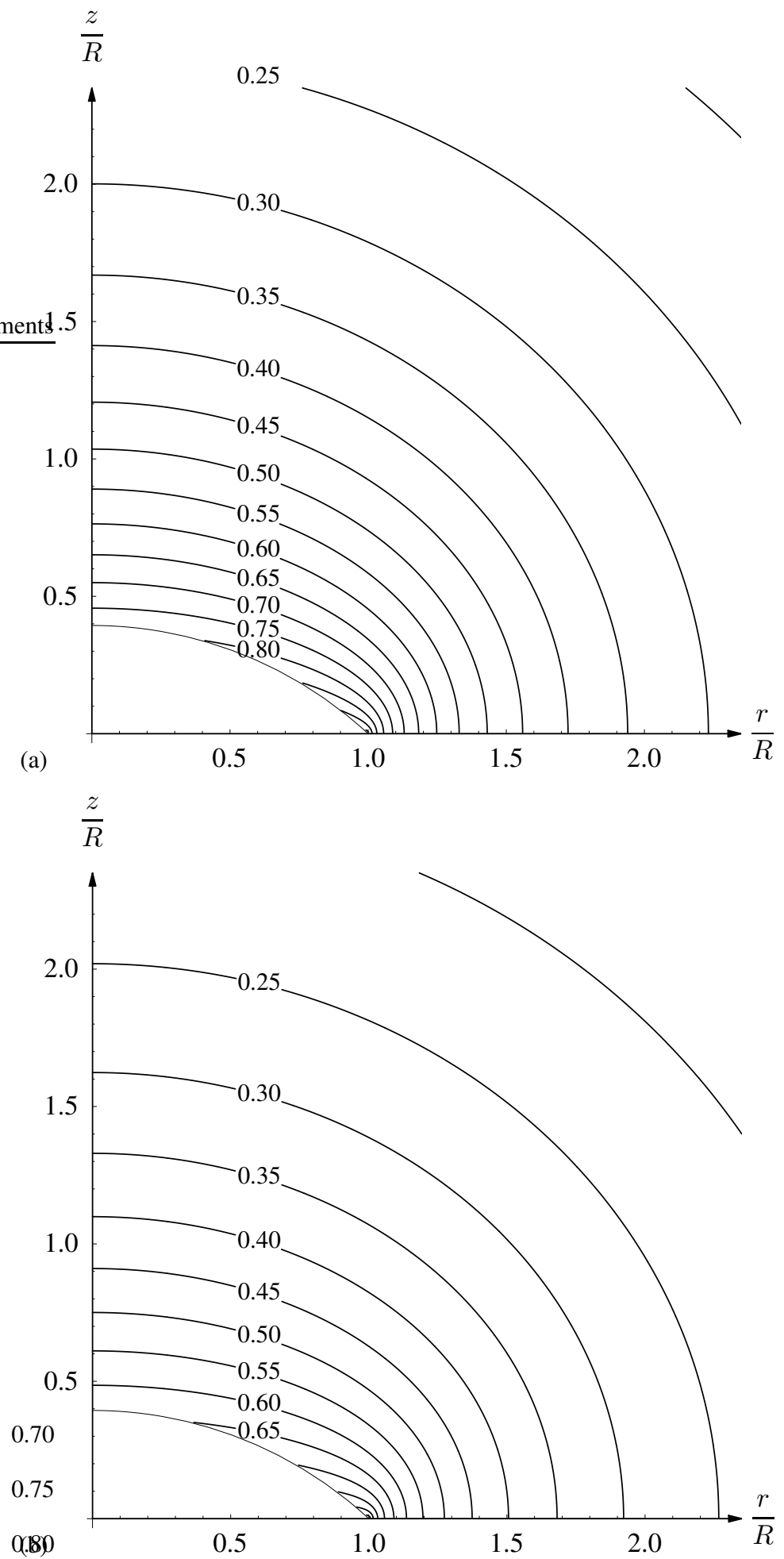

FIGURE 10. Theoretical predictions of the present model for the contours of the initial concentration of vapour in the atmosphere for a droplet of methanol of radius $R=1.35 \mathrm{~mm}$ on (a) an Al and (b) a PTFE substrate. The contours are labelled with the appropriate values of $c / c_{\mathrm{sat}}\left(T_{\mathrm{a}}\right)$. 


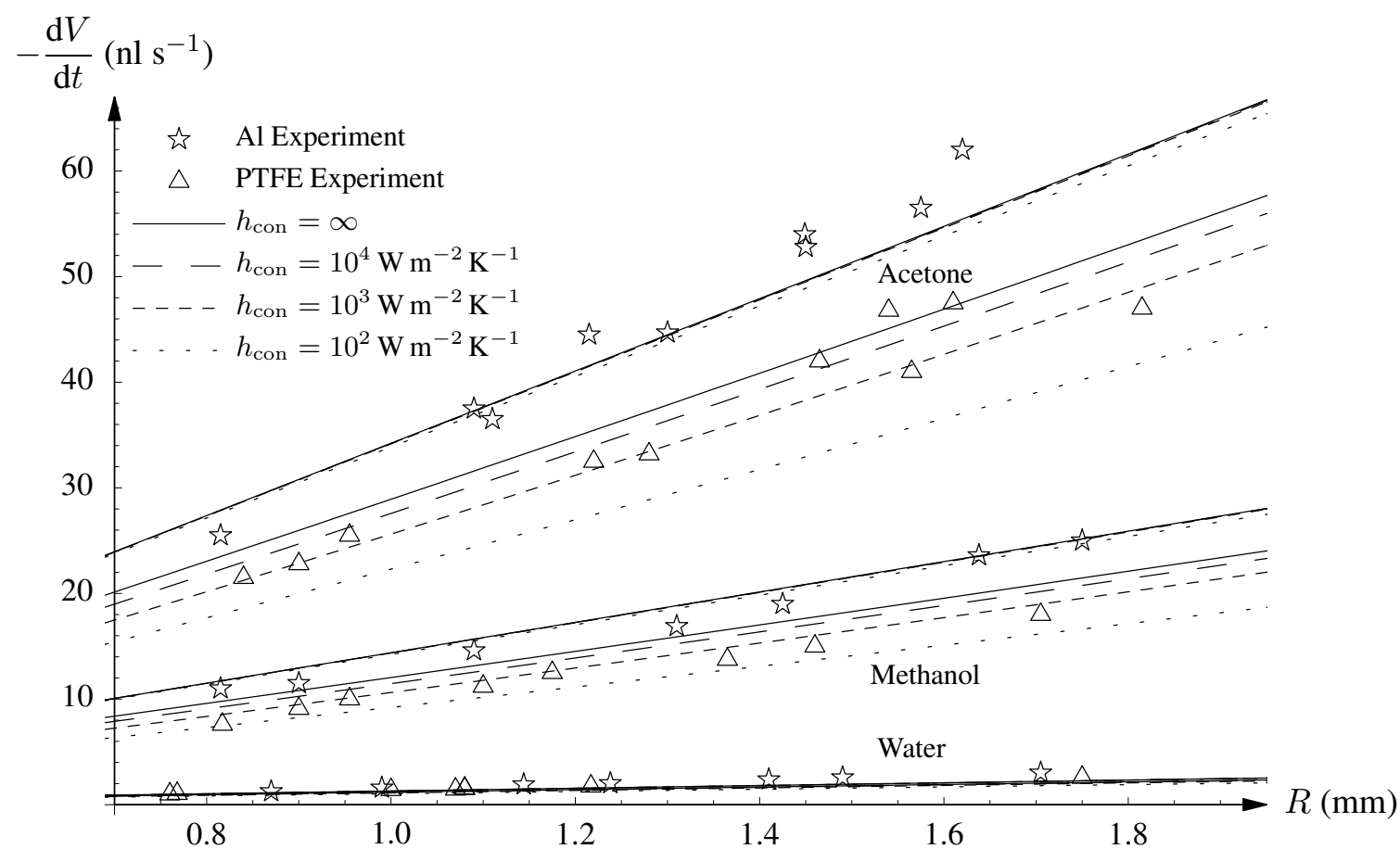

FIGURE 11. Comparison between experimentally measured and theoretically predicted values of the average total evaporation rate plotted as a function of droplet radius $R$ for droplets of acetone, methanol and water on $\mathrm{Al}$ and PTFE substrates including the theoretical predictions for the improved model with a Newton's law of cooling with convective heat transfer coefficients $h_{\text {con }}=\infty, 10^{4}, 10^{3}$ and $10^{2} \mathrm{~W} \mathrm{~m}^{-2} \mathrm{~K}^{-1}$.

\section{Improvements to the Mathematical Model}

As we have already seen, Figures 5 and 6 show that the theoretical predictions of the present model for the average total evaporation rate for droplets of acetone and methanol are in good quantitative agreement with the experimental results, while both the basic model and the present model systematically underpredict the experimental results for droplets of water. In this section we consider two ad hoc improvements to the present model, namely including a Newton's law of cooling on the unwetted surface of the substrate and the buoyancy of water vapour in the atmosphere.

\subsection{Newton's Law of Cooling}

As we have already seen, the present model slightly over-predicts the average total evaporation rate for a droplet of acetone or methanol on a PTFE substrate. We can improve the agreement by replacing equation 


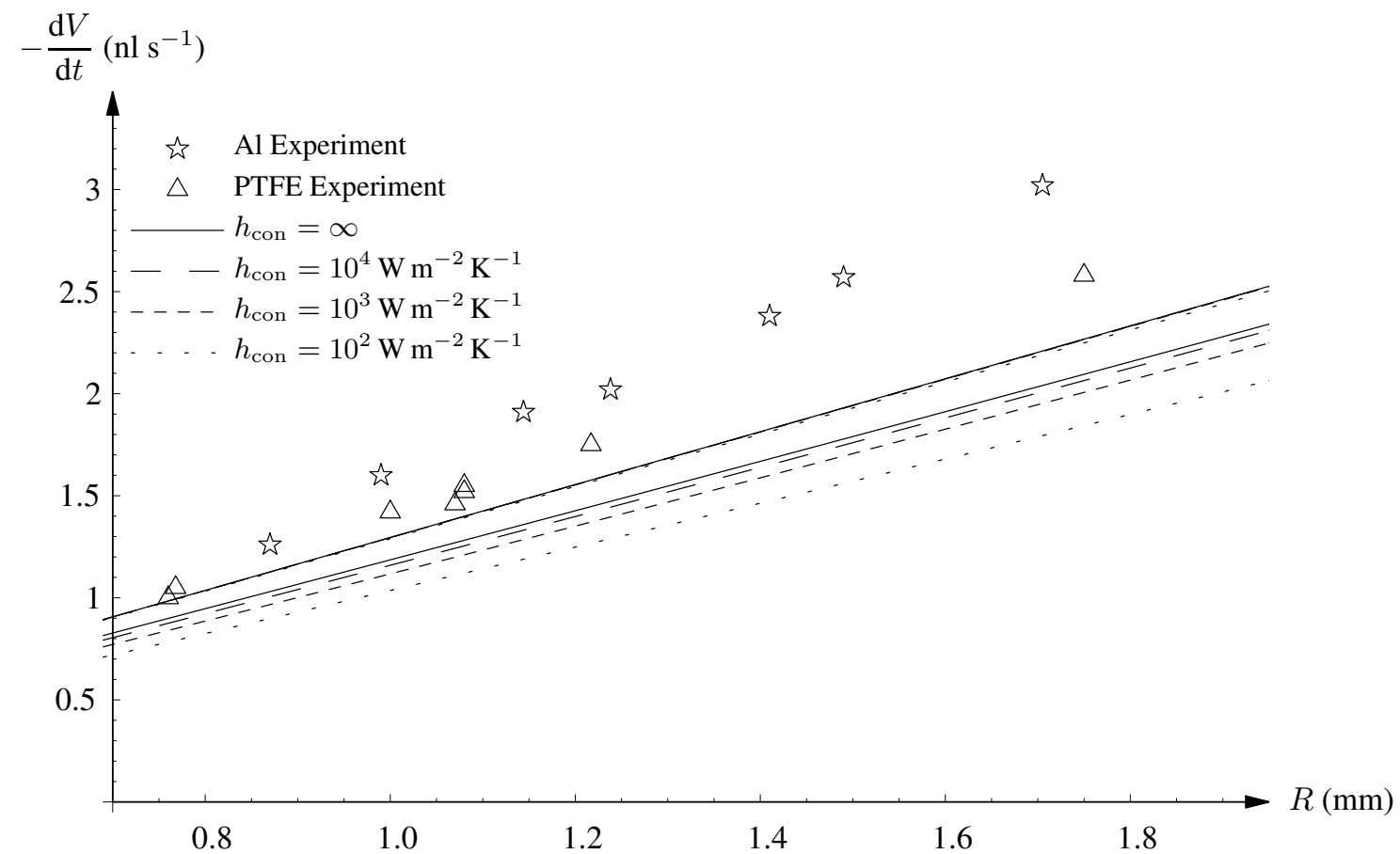

FIGURE 12. An enlargement of the lower part of Figure 11 showing the results for droplets of water in more detail.

(3.8) with an ad hoc "Newton's law of cooling" in the form

$$
T^{\mathrm{s}}=T_{\mathrm{a}}-\frac{k^{\mathrm{s}}}{h_{\mathrm{con}}} \frac{\partial T^{\mathrm{s}}}{\partial n}
$$

on the unwetted surface of the substrate (i.e. on $z=0$ for $r>R$ and on $z=-h^{\mathrm{s}}$ ), where $h_{\text {con }}$ is an empirical convective heat transfer coefficient and $n$ is an outward coordinate to the substrate.

Figures 11 and 12 are improved versions of Figures 5 and 6 including the theoretical predictions for the average total evaporation rate for droplets of acetone, methanol and water with a Newton's law of cooling for a range of values of $h_{\text {con }}$ showing the sensitivity of the results to the value of $h_{\text {con }}$. In addition, Table 4 includes the corresponding theoretical predictions for the initial temperature in the bulk of the droplet of radius $R=1.35 \mathrm{~mm}$ of all three liquids on all four substrates with $h_{\text {con }}=10^{3} \mathrm{~W} \mathrm{~m}^{-2} \mathrm{~K}^{-1}$. Since, as we have already seen, an $\mathrm{Al}$ substrate is essentially at the constant atmospheric temperature $T_{\mathrm{a}}$ we would not expect the improved thermal boundary condition to have any significant effect, and this is confirmed by the results shown in Table 4 and Figure 11, which show that there is essentially no change in either the 
evaporative cooling or the average total evaporation rate compared to the original version of the present model (i.e. the case $h_{\text {con }}=\infty$ ). However, for a PTFE substrate we would expect the improved boundary condition to have a significant effect, and this too is confirmed by the results shown in Table 4 and Figure 11 , which show that there is an increase in the evaporative cooling and a decrease in the average total evaporation rate compared to the original version of the present model. In particular, choosing the value $h_{\text {con }}=10^{3} \mathrm{~W} \mathrm{~m}^{-2} \mathrm{~K}^{-1}$ gives excellent quantitative agreement for droplets of acetone and methanol on a PTFE substrate, suggesting that Newton cooling on the unwetted surface of the substrate may indeed be significant. However, as Figure 12 shows, the improved model still systematically under-predicts the experimental results for droplets of water.

Figure 13 is an improved version of Figure 9 showing the theoretical predictions for the initial temperature contours in the $r, z$-plane with a Newton's law of cooling with $h_{\text {con }}=10^{3} \mathrm{~W} \mathrm{~m}^{-2} \mathrm{~K}^{-1}$. In particular, comparing Figures 9 and 13 shows that the effect of the improved boundary condition is to increase the evaporative cooling on a PTFE (but not significantly on an Al) substrate.

\subsection{Buoyancy of Water Vapour in the Atmosphere}

As we have already described, Shahidzadeh-Bonn et al. (2006) and Guéna, Poulard \& Cazabat (2007a) came to different conclusions about the significance of buoyant convection of water vapour in the atmosphere in their experiments. Computing the velocity of the air/vapour mixture (as done by, for example, Savino, Paterna \& Favaloro (2002) for a similar problem) is a significant computational task which we do not attempt here. Simple dimensional considerations indicate that the dominant balance is between buoyancy and inertia (rather than viscous) effects and hence that the upward velocity of the air/vapour mixture will be of the order of

$$
u_{0}=\left(\frac{\left(\rho^{\mathrm{a}}-\rho^{\mathrm{m}}\right) g L}{\rho^{\mathrm{m}}}\right)^{\frac{1}{2}}
$$




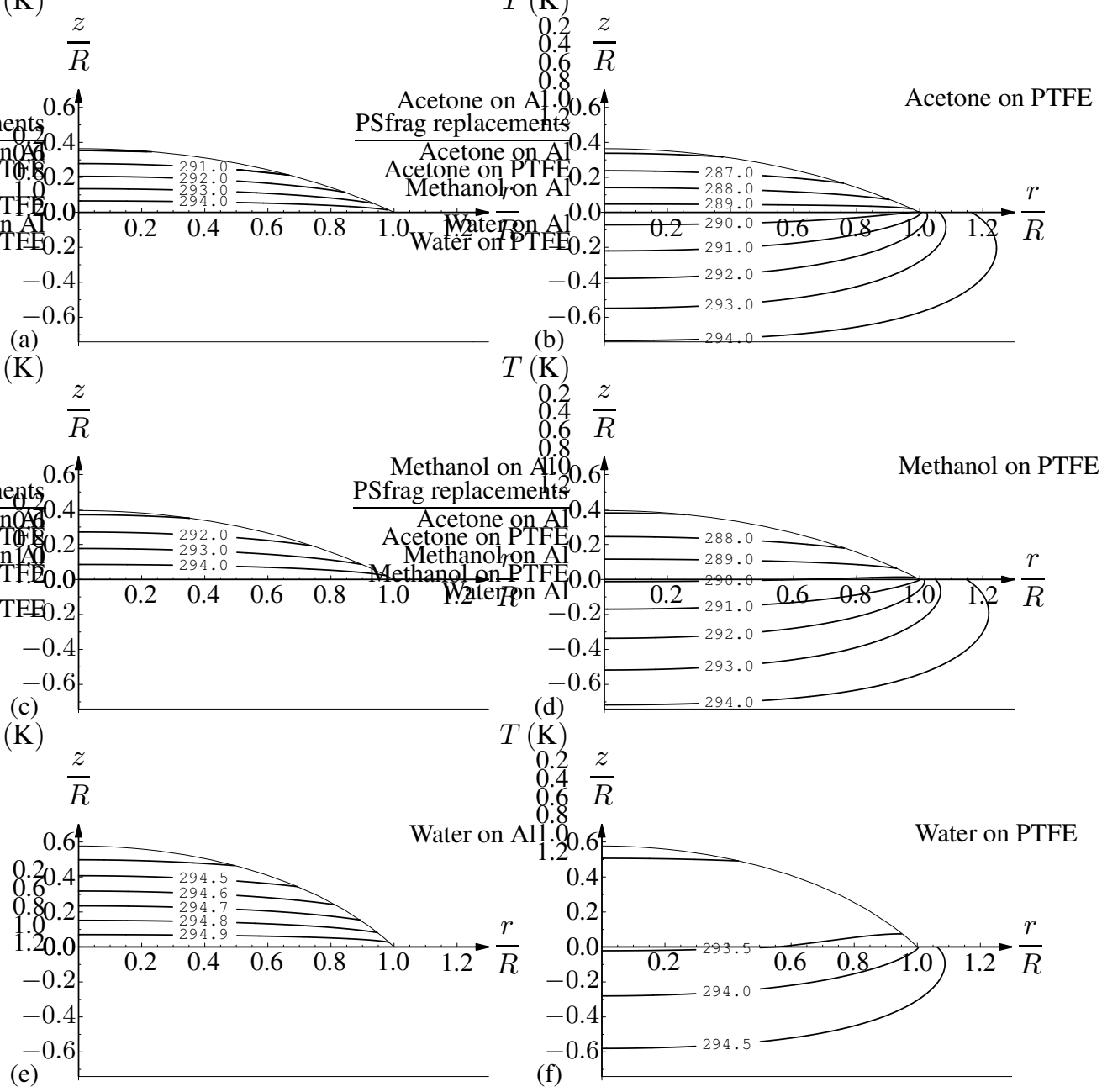

FIGURE 13. Theoretical predictions of the present model for the initial temperature contours in the $r, z$-plane within the substrate and a droplet of $(\mathrm{a}, \mathrm{b})$ acetone, $(\mathrm{c}, \mathrm{d})$ methanol and (e,f) water of radius $R=1.35 \mathrm{~mm}$ on (a,c,e) $\mathrm{Al}$ and (b,d,f) PTFE substrates including the theoretical predictions for the improved model with a Newton's law of cooling with convective heat transfer coefficient $h_{\text {con }}=10^{3} \mathrm{~W} \mathrm{~m}^{-2} \mathrm{~K}^{-1}$.

(as given by, for example, Shahidzadeh-Bonn et al. (2006)), where $\rho^{\mathrm{a}}, \rho^{\mathrm{v}}$ and

$$
\rho^{\mathrm{m}}=\rho^{\mathrm{a}}-\left(\frac{\rho^{\mathrm{a}}-\rho^{\mathrm{v}}}{\rho^{\mathrm{v}}}\right) c
$$

are the density of the air, the vapour and the air/vapour mixture, respectively, and $L$ is a characteristic lengthscale of the flow in the atmosphere. Taking $\rho^{\mathrm{a}}=1.293 \mathrm{~kg} \mathrm{~m}^{-3}, \rho^{\mathrm{v}}=0.800 \mathrm{~kg} \mathrm{~m}^{-3}$ from Tennent (1971) and approximating $c \simeq c_{\text {sat }}\left(T_{\mathrm{a}}\right)=1.94 \times 10^{-2} \mathrm{~kg} \mathrm{~m}^{-3}$ leads to $\left(\rho^{\mathrm{a}}-\rho^{\mathrm{m}}\right) / \rho^{\mathrm{a}} \simeq 0.01$, in agreement with the value given by Shahidzadeh-Bonn et al. (2006), but whereas Shahidzadeh-Bonn et 


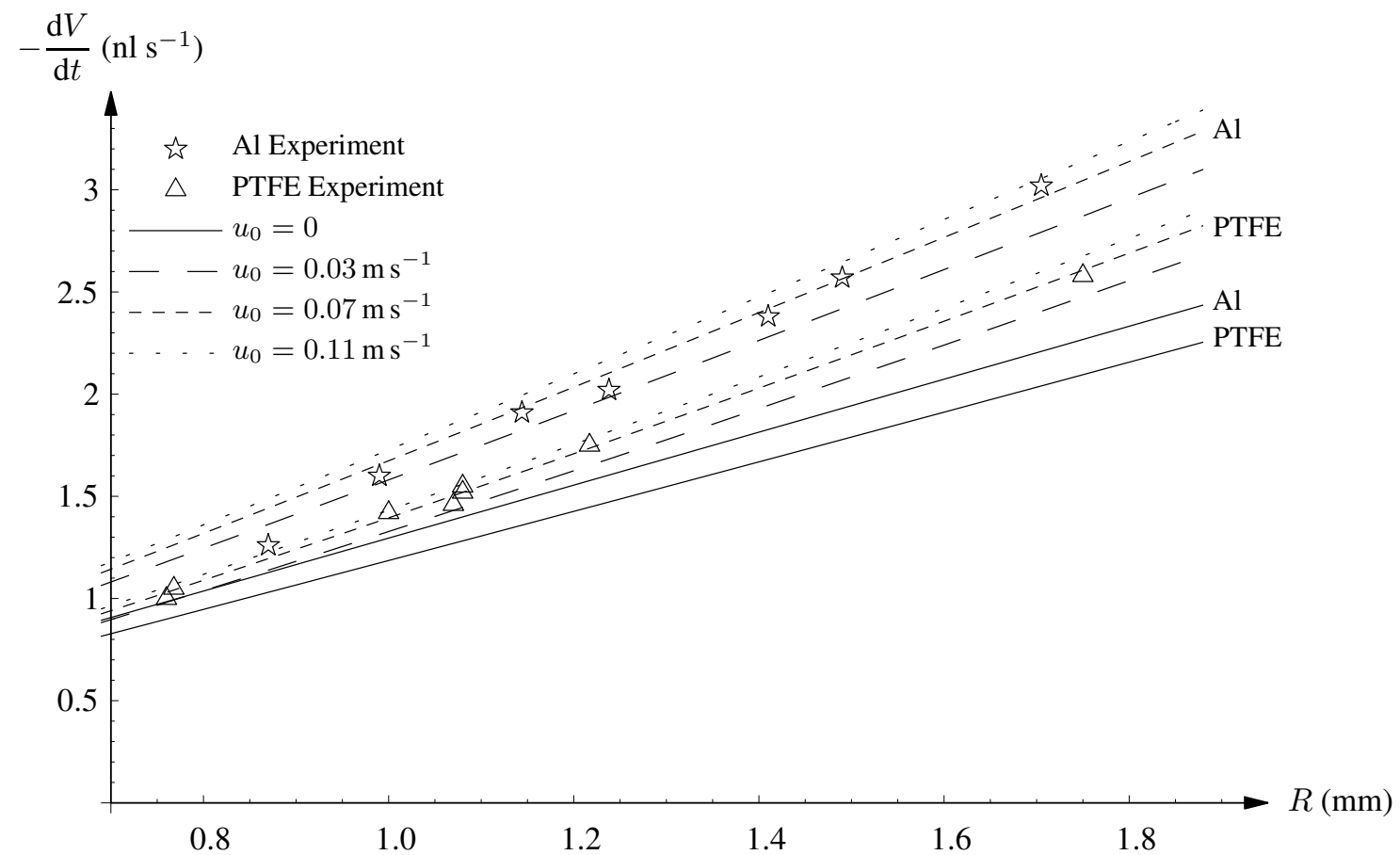

FIGURE 14. Comparison between experimentally measured and theoretically predicted values of the average total evaporation rate plotted as a function of droplet radius $R$ for droplets of water on $\mathrm{Al}$ and PTFE substrates including the theoretical predictions with a Newton's law of cooling with convective heat transfer coefficient $h_{\mathrm{con}}=10^{3} \mathrm{~W} \mathrm{~m}^{-2} \mathrm{~K}^{-1}$ and buoyant convection with constant buoyant velocity $u_{0}=0,0.03,0.07$ and $0.11 \mathrm{~m} \mathrm{~s}^{-1}$.

al. (2006) took $L=10^{-3} \mathrm{~m}$ (corresponding to the typical lengthscale of their droplets) and estimated $u_{0}=0.01 \mathrm{~m} \mathrm{~s}^{-1}$, we take $L=10^{-1} \mathrm{~m}$ (corresponding to a more realistic estimate of the lengthscale of the flow in the atmosphere) and estimate $u_{0}=0.1 \mathrm{~m} \mathrm{~s}^{-1}$. In order to investigate the possibility of buoyant convection of water vapour as an explanation for the systematic under-prediction of the experimental results for droplets of water in the present experiments we replace Laplace's equation (3.9) with a quasisteady convection-diffusion equation in the form

$$
D \nabla^{2} c=\mathbf{u} \cdot \nabla c
$$

and the local mass flux from the free surface of the droplet (3.13) with

$$
J=-D \nabla c \cdot \mathbf{n}+(\mathbf{n} \cdot \mathbf{u}) c
$$


on $z=h$ for $r<R$, where $\mathbf{u}=\mathbf{u}(r, z, t)$ is the velocity of the atmosphere/vapour mixture, which we estimate in a simple ad hoc manner by taking $\mathbf{u}=\left(0,0, u_{0}\right)$, where the constant buoyant velocity $u_{0}$ is given by (6.2).

Figure 14 is an improved version of Figure 6 including the theoretical predictions for the average total evaporation rate for a droplet of water with a Newton's law of cooling with $h_{\text {con }}=10^{3} \mathrm{~W} \mathrm{~m}^{-2} \mathrm{~K}^{-1}$ and buoyant convection for a range of values of the constant buoyant velocity $u_{0}$ showing the sensitivity of the results to the value of $u_{0}$. In addition, Table 4 includes the corresponding theoretical predictions for the initial temperature in the bulk of droplets of radius $R=1.35 \mathrm{~mm}$ of all three liquids on all four substrates with $h_{\text {con }}=10^{3} \mathrm{~W} \mathrm{~m}^{-2} \mathrm{~K}^{-1}$ and $u_{0}=0.07 \mathrm{~m} \mathrm{~s}^{-1}$. In particular, Figure 14 shows that choosing the physically realistic value $u_{0}=0.07 \mathrm{~m} \mathrm{~s}^{-1}$ gives excellent quantitative agreement for droplets of water on both $\mathrm{Al}$ and PTFE substrates, suggesting that buoyant convection of water vapour may indeed play a significant role in enhancing the diffusion of vapour away from a droplet of water in the present experiments.

\section{Conclusions}

In the present work we reported the results of physical experiments that demonstrate the strong influence of the thermal conductivity of the substrate on the evaporation of a pinned droplet. We showed that this behaviour can be captured by a mathematical model including the variation of the saturation concentration with temperature, and hence coupling the problems for the vapour concentration in the atmosphere and the temperature in the liquid and the substrate. In section 5 we showed that the resulting theoretical predictions for the average evaporation rate of droplets of acetone and methanol are in good quantitative agreement with the experimental results, while both the basic model and the present model systematically under-predict the experimental results for droplets of water. In section 6 we showed that including two ad hoc improvements to the model, namely a Newton's law of cooling on the unwetted surface of the substrate 
and the buoyancy of water vapour in the atmosphere, gives excellent quantitative agreement for all three liquids on both $\mathrm{Al}$ and PTFE substrates. In particular, although direct measurements of the flow within the droplets were not made, the good agreement between the present experimentally measured and theoretically predicted values suggests that thermocapillary effects are probably not significant in the present experiments.

Clearly despite this success there is still much theoretical work to do to construct and analyse mathematical models for more complicated situations, including the influence of using different ambient gases and of varying the atmospheric pressure, of using droplets of binary mixtures liquids, and of using structured substrates, investigated experimentally by David (2007). Dunn et al. (2008) incorporated the first two of these effects into their model for the evaporation of a thin droplet on a thin substrate, but generalising this to the larger contact angles observed experimentally and the study of the evaporation of binary drops (perhaps building on the work of Howison et al. (1997) on an evaporating layer of paint consisting of a volatile solvent and a non-volatile resin) remain interesting open issues.

\section{Acknowledgement}

This work was supported by the United Kingdom Engineering and Physical Sciences Research Council via joint grants GR/S59444 (Edinburgh) and GR/S59451 (Strathclyde).

\section{REFERENCES}

Anderson, D. M. \& DAVIS, S. H. 1995 The spreading of volatile liquid droplets on heated surfaces. Phys. Fluids 7, $248-265$. AJAEV, V. S. 2005 Spreading of thin volatile liquid droplets on uniformly heated surfaces. J. Fluid Mech. 528, 279-296.

Bernardin, J. D., Mudawar, I., Walsh, C. B. \& Franses, E. I. 1997 Contact angle temperature dependence for water droplets on practical aluminium surfaces. Int. J. Heat Mass Transfer 40, 1017-1033.

BIRDi, K. S., VU, D. T. \& WinTER, A. 1989 A study of the evaporation rates of small water drops placed on a solid surface. J. Phys. Chem. 93, 3702-3703.

Bourgès-Monnier, C. \& SHANAhan, M. E. R. 1995 Influence of evaporation on contact angle. Langmuir 11, $2820-2829$. 
Burelbach, J. P., Bankoff, S. G. \& Davis, S. H. 1988 Nonlinear stability of evaporating/condensing liquid films. J. Fluid Mech. 195, 463-494.

Cachile, M., BÉnichou, O. \& CAZABAt, A. M. 2002 Evaporating droplets of completely wetting liquids. Langmuir 18, 7985-7990.

Cachile, M., BÉnichou, O., Poulard, C. \& Cazabat, A. M. 2002 Evaporating droplets. Langmuir 18, 8070-8078.

Chandra S., Di Marzo M., Qiao Y. M. \& TARTARini P. 1996 Effect of liquid-solid contact angle on droplet evaporation. Fire Safety Journal 27, 141-158.

COMSOL Multiphysics website www. comsol . com accessed on 25th October 2007.

Corning Incorporated website www. corning . com accessed on 19th November 2007.

CRAFTON, E. F. \& BLACK, W. Z. 2004 Heat transfer and evaporation rates of small liquid droplets on heated horizontal surfaces. Int. J. Heat Mass Transfer 47, 1187-1200.

DAVID, S. 2007 An investigation of the wetting behaviour of evaporative drops, Ph. D. Thesis, University of Edinburgh.

David, S., Sefiane, K. \& TAdrist, L. 2007 Experimental investigation of the effect of thermal properties of the substrate in the wetting and evaporation of sessile drops. Colloids and Surfaces A: Physiochem. Eng. Aspects 298, 108-114.

Deegan, R. D., Bakajin, O., Dupont, T. F., Huber, G., Nagel, S. R. \& Witten, T. A. 1997 Capillary flow as the cause of ring stains from dried liquid drops. Nature 389, 827-829.

Deegan, R. D., Bakajin, O., Dupont, T. F., Huber, G., Nagel, S. R. \& Witten, T. A. 2000 Contact line deposits in an evaporating drop. Phys. Rev. E 62, 756-765.

Dunn, G. J., Wilson, S. K., Duffy, B. R., David, S. \& Sefiane, K. 2008 A mathematical model for the evaporation of a thin sessile liquid droplet: comparison between experiment and theory. Colloids and Surfaces A: Physiochem. Eng. Aspects 323, 50-55.

Erbil, H. Y., Mchale, G. \& Newton, M. I. 2002a Analysis of evaporating thick liquid films on solids. J. Adhesion Science Technol. 16, 1869-1881.

Erbil, H. Y., McHale, G. \& Newton, M. I. 2002b Drop evaporation on solid surfaces: constant contact angle mode. Langmuir 18, 2636-2641.

FAng, G. AND WARD, C. A. 1999 Temperature measured close to the interface of an evaporating liquid. Phys. Rev. E 59, $417-428$.

Grandas, L., REynARD, C., SANTINI, R. \& TADRIST, L. 2005 Experimental study of the evaporation of a sessile drop on a heated wall. Wetting influence. Int. J. Therm. Sci. 44, 137-146. 
Guéna, G., Poulard, C., Voué, M., De Coninck, J. \& Cazabat, A. M. 2006 Evaporation of sessile liquid droplets. Colloids and Surfaces A: Physiochem. Eng. Aspects 291, 191-196.

GuÉnA, G., AllançOn, P. \& CAZABAt, A. M. 2007 Receding contact angle in the situation of complete wetting: experimental check of a model used for evaporating droplets. Colloids and Surfaces A: Physiochem. Eng. Aspects 300, 307-314.

GuÉna, G., Poulard, C. \& CaZabat, A. M. 2007a The leading edge of evaporating droplets. J. Coll. Int. Sci. 312, $164-171$.

Guéna, G., Poulard, C. \& Cazabat, A. M. 2007b Evaporating drops of alkane mixtures. Colloids and Surfaces A: Physiochem. Eng. Aspects 298, 2-11.

Howison, S. D., Moriarty, J. A., Ockendon, J. R., Terrill, E. L. \& Wilson, S. K. 1997 A mathematical model for drying paint layers. J. Eng. Maths 32, 377-394.

Hu, H. \& LARSON, R. G. 2002 Evaporation of a sessile droplet on a substrate. J. Phys. Chem. B 106, 1334-1344.

HU, H. \& LARSON, R. G. 2005a Analysis of the microfluid flow in an evaporating sessile droplet. Langmuir 21, 3963-3971.

HU, H. \& LARSON, R. G. 2005b Analysis of the effects of Marangoni stresses on the microflow in an evaporating sessile droplet. Langmuir 21, 3972-3980.

Hu, H. \& LARSOn, R. G. 2006 Marangoni effect reverses coffee-ring depositions. J. Phys. Chem. B 110, 7090-7094.

Lebedev, N. N. 1965 Special Functions and Their Applications. Prentice-Hall.

Lide, D. R. \& KeHIAIAN, H. V. 1994 CRC Handbook of Thermophysical and Thermochemical Data. CRC Press.

LUGG, A. G. 1968 Diffusion coefficients of some organic and other vapors in air. Analytical Chemistry 40, 1072-1077.

Mollaret, R., Sefiane, K., Christy, J. R. E. \& Veyret, D. 2004 Experimental and numerical investigation of the evaporation into air of a drop on a heated surface. Trans. IChemE, Part A 82, 471-480.

Monteith, J. L. 1973 Principles of Environmental Physics. Edward Arnold.

Panwar, A. K., BARthwal, S. K. \& Ray, S. 2003 Effect of evaporation on the contact angle of a sessile drop on solid substrates. J. Adhesion Science Technol. 17, 1321-1329.

Perry, R. H. \& Green, D. W. 1997 Perry's Chemical Engineers' Handbook, 7th edn. McGraw-Hill.

Picknett, R. G. \& BEXON, R. 1977 The evaporation of sessile or pendant drops in still air. J. Coll. Int. Sci. 61, 336-350.

Popov, Y. O. 2005 Evaporative deposition patterns: spatial dimensions of the deposit. Phys. Rev. E 71, 036313-1-036313-17.

Poulard, C., BÉnichou, O. \& CAZABAt, A. M. 2003 Freely receding evaporating droplets. Langmuir 19, 8828-8834.

Poulard, C., Guéna, G., Cazabat, A. M., Boudaoud, A. \& Ben Amar, M. 2005 Rescaling the dynamics of evaporating drops. Langmuir, 21, 8226-8233.

Poulard, C., Guéna, G. \& Cazabat, A. M. 2005 Diffusion-driven evaporation of sessile drops. J. Phys.: Condens. Matter 17, S4213-S4227. 
RAZNJEVIC, K. 1995 Handbook of Thermodynamic Tables. Begell House.

Reid, R. C., Prausnitz, J. M. \& Poling, B. E. 1987 The Properties of Gases \& Liquids, 4th edn. McGraw-Hill.

Ristenpart, W. D., Kim, P. G., Domingues, C., Wan, J. \& Stone, H. A. 2007 Influence of substrate conductivity on circulation reversal in evaporating drops. Phys. Rev. Lett. 99, 234504-1-234502-4. Supplementary material EPAPS document number E-PRLTAO-99-072744 available online via www. aip.org/pubservs/epaps.html accessed on 25th April 2008.

Rowan, S. M., Newton, M. I. \& McHale, G. 1995 Evaporation of microdroplets and the wetting of solid surfaces. J. Phys. Chem. 99, 13268-13271.

SaVino, R., Paterna, D. \& FaVAloro, N. 2002 Buoyancy and Marangoni effects in an evaporating drop. J. Thermophys. Heat Trans. 16, 562-574.

SEFIANE, K. \& TADRIST, L. 2006 Experimental investigation of the de-pinning phenomenon on rough surfaces of volatile drops. Int. Comm. Heat Mass Trans. 33, 482-490.

Shahidzadeh-Bonn, N., Rafaï, S., Azouni, A. \& Bonn, D. 2006 Evaporating droplets. J. Fluid Mech. 549, $307-313$.

Shanahan, M. E. R. 2001a Condensation transport in dynamic wetting. Langmuir 17, 3997-4002.

SHANAHAN, M. E. R. 2001b Spreading of water: condensation effects. Langmuir 17, 8229-8235.

SodtKe, C., AjAev, V. S. \& Stephan, P. 2007 Evaporation of thin liquid droplets on heated surfaces. Heat Mass Transfer 43, $649-657$.

Sultan, E., Boudaoud, A. \& Ben Amar, M. 2005 Evaporation of a thin film: diffusion of the vapour and Marangoni instabilities. J. Fluid Mech. 543, 183-202.

TAnaka, K., Fujita, I. \& Uematsu, M. 2007 Isobaric specific heat capacity of $\left\{x \mathrm{CH}_{3} \mathrm{OH}+(1-x) \mathrm{H}_{2} \mathrm{O}\right\}$ with $x=(1.0000$, $0.7943,0.4949,0.2606,0.1936,0.1010$, and 0.0496$)$ at $T=(280,320$, and 360$) \mathrm{K}$ in the pressure range from $(0.1$ to 15$)$ MPa. J. Chem. Thermodynamics 39, 961-966.

Tennent, R. M. 1971 Science Data Book. Oliver and Boyd.

XU, X. \& LuO, J. 2007 Marangoni flow in an evaporating water droplet. Appl. Phys. Lett. 91, 124102-1-124102-3. 